Ann. Inst. Henri Poincaré,

Vol. $12, \mathrm{n}^{\circ} 4,1995$, p. $459-503$.

Analyse non linéaire

\title{
Normal form and global solutions for the Klein-Gordon-Zakharov equations
}

\author{
by \\ T. OZAWA ${ }^{\dagger}$, K. TSUTAYA ${ }^{\dagger}$ \\ ${ }^{\dagger}$ Department of Mathematics \\ Hokkaido University \\ Sapporo 060, Japan \\ and \\ Y. TSUTSUMI ${ }^{\ddagger}$ \\ ${ }^{\ddagger}$ Department of Mathematical Sciences \\ University of Tokyo \\ Hongo, Tokyo 113, Japan
}

\begin{abstract}
In this paper we study the global existence and asymptotic behavior of solutions for the Cauchy problem of the Klein-Gordon-Zakharov equations in three space dimensions. We prove that for small initial data, there exist the unique global solutions of the Klein-Gordon-Zakharov equations. We also show that these solutions approach asymptotically the free solutions as $t \rightarrow \infty$. Our proof is based on the method of normal forms introduced by Shatah [12], which transforms the original system with quadratic nonlinearity into a new system with cubic nonlinearity.
\end{abstract}

RÉSumÉ. - Dans cet article, nous étudions l'existence globale et le comportement asymptotique de solutions pour le problème de Cauchy des équations de Klein-Gordon-Zakharov en trois dimensions. Nous montrons que pour des petites données initiales, il existe les solutions globales uniques des équations de Klein-Gordon-Zakharov. Nous montrons aussi que ces solutions approchent des solutions libres asymptotiquement lorsque $t \rightarrow \infty$. Notre preuve est basée sur la méthode de formes normales introduite par Shatah [12], qui transforme le système original avec non-linéarité quadratique en un système neuf avec non-linéarité cubique. 


\section{INTRODUCTION AND MAIN RESULTS}

In the present paper we consider the Cauchy problem of the KleinGordon-Zakharov equations in three space dimensions:

$$
\begin{aligned}
& \partial_{t}^{2} u-\Delta u+u=-n u, \quad t>0, \quad x \in \mathbf{R}^{3}, \\
& \partial_{t}^{2} n-\Delta n=\Delta|u|^{2}, \quad t>0, \quad x \in \mathbf{R}^{3}, \\
& u(0, x)=u_{0}(x), \quad \partial_{t} u(0, x)=u_{1}(x), \\
& n(0, x)=n_{0}(x), \quad \partial_{t} n(0, x)=n_{1}(x),
\end{aligned}
$$

where $\partial_{t}=\partial / \partial t$, and $u(t, x)$ and $n(t, x)$ are functions from $\mathbf{R}_{+} \times \mathbf{R}^{3}$ to $\mathbf{C}^{3}$ and from $\mathbf{R}_{+} \times \mathbf{R}^{3}$ to $\mathbf{R}$, respectively. The system (1.1)-(1.2) describes the propagation of strong turbulence of the Langmuir wave in a high frequency plasma (see [15]). The usual Zakharov system

$$
\begin{aligned}
& i \partial_{t} u+\Delta u=n u, \quad t>0, \quad x \in \mathbf{R}^{3}, \\
& \partial_{t}^{2} n-\Delta n=\Delta|u|^{2}, \quad t>0, \quad x \in \mathbf{R}^{3}
\end{aligned}
$$

is derived from (1.1)-(1.2) through the physical approximation procedure.

In the present paper we consider solving (1.1)-(1.3) around the zero solutions. There are many papers concerning the global existence of small solutions for the coupled systems of the Klein-Gordon and wave equations with quadratic nonlinearity (see, e.g., [1], [5]-[7], [9], [10], [12] and [13]). The methods to solve those systems can be classified into two groups (for a good review of this matter, see Strauss [14]). One is to use the Sobolev space with weight related to the generators of the Lorentz group. This was developed by Klainerman [9] and [10]. The combination of this method and the null condition technique has produced several nice applications to the hyperbolic systems of physical importance (see, e.g., Bachelot [1] and Georgiev [6]). However, this method does not seem to be directly applicable to (1.1)-(1.3). In fact, since the system (1.1)-(1.2) consists of the Klein-Gordon and wave equations with quadratic nonlinearity in three space dimensions, we need to use not only the Sobolev norms with weights related to the generators of the Lorentz group but also the null condition technique (see, e.g., Georgiev [5] and [6]). But the nonlinear terms in (1.1) and (1.2) do not seem to satisfy the null condition as they are. Another method is based on the theory of normal forms introduced by Shatah [12], which is an extension of Poincaré's theory of normal forms to the partial 
differential equations. See also [16] and its references. In this paper we apply the argument of normal form to (1.1)-(1.2) and prove the global existence of solutions to (1.1)-(1.3) for small initial data. We also show that these global solutions to (1.1)-(1.3) with small initial data approach the free solutions asymptotically as $t \rightarrow+\infty$.

Before we state the main results in this paper, we give several notations. For $1 \leq p \leq \infty$ and a nonnegative integer $m$, let $L^{p}$ and $W^{m, p}$ denote the standard $L^{p}$ and Sobolev spaces on $\mathbf{R}^{3}$, respectively. We put $H^{m}=W^{m, 2}$. For a positive integer $m$, we denote the dual space of $H^{m}$ by $H^{-m}$. For $s \in \mathbf{R}$ and $1<p<\infty$, we let $\dot{H}^{s, p}$ be the completion of all functions $v \in \mathcal{S}$ with $0 \notin \operatorname{supp} \hat{v}$ with respect to the semi-norm $\|v\|_{\dot{H}^{s, p}} \equiv\left\|(-\Delta)^{s / 2} v\right\|_{L^{p}}$, where $\mathcal{S}$ is the Schwartz space on $\mathbf{R}^{3}$ and $\hat{v}$ denotes the Fourier transform of $v$. We write $\dot{H}^{s}=\dot{H}^{s, 2}$. We put $\omega=(1-\Delta)^{1 / 2}$ and $\omega_{0}=(-\Delta)^{1 / 2}$.

We have the following theorem concerning the global existence and asymptotic behavior of solutions to (1.1)-(1.3) for small initial data.

TheOREM 1.1. - Let $0<\varepsilon \leq 10^{-2}$. Assume that $u_{0} \in H^{52} \cap W^{29,6 /(5+2 \varepsilon)}$, $u_{1} \in H^{51} \cap W^{28,6 /(5+2 \varepsilon)}, n_{0} \in H^{51} \cap W^{28,220 / 217} \cap \dot{H}^{-1}$ and $n_{1} \in$ $H^{50} \cap W^{27,220 / 217} \cap \dot{H}^{-2}$. Then, there exists a $\delta>0$ such that if

$$
\begin{aligned}
& \left\|u_{0}\right\|_{H^{52} \cap W^{29,6 /(5+2 \varepsilon)}}+\left\|u_{1}\right\|_{H^{51} \cap W^{28,6 /(5+2 \varepsilon)}} \\
& \quad+\left\|n_{0}\right\|_{H^{51} \cap W^{28,220 / 217} \cap \dot{H}^{-1}}+\left\|n_{1}\right\|_{H^{50} \cap W^{27,220 / 217} \cap \dot{H}^{-2}} \leq \delta
\end{aligned}
$$

(1.1)-(1.3) have the unique global solutions $(u, n)$ satisfying

$$
u \in \bigcap_{j=0}^{2} C^{j}\left([0, \infty) ; H^{52-j}\right)
$$

$$
\begin{array}{r}
n \in\left[\bigcap_{j=0}^{2} C^{j}\left([0, \infty) ; H^{51-j}\right)\right] \\
\bigcap\left[\bigcap_{j=0}^{1} C^{j}\left([0, \infty) ; \dot{H}^{-1-j}\right)\right],
\end{array}
$$

$$
\sum_{j=0}^{1}\left\|\partial_{t}^{j} u(t)\right\|_{W^{26-j, 6 /(1-2 \varepsilon)}}=O\left(t^{-(1+\varepsilon)}\right) \quad(t \rightarrow \infty)
$$

$$
\sum_{j=0}^{1}\left\|\partial_{t}^{j} n(t)\right\|_{W^{25-j, 220 / 3}}=O\left(t^{-107 / 110}\right) \quad(t \rightarrow \infty),
$$


where $\delta$ depends only on $\varepsilon$. Furthermore, the above solutions $(u, n)$ of (1.1)-(1.3) have the free profiles $u_{+0} \in H^{52}, u_{+1} \in H^{51}, n_{+0} \in H^{51}$ and $n_{+1} \in H^{50}$ such that

$$
\begin{aligned}
& \sum_{j=0}^{1}\left\|\partial_{t}^{j}\left(u(t)-u_{+}(t)\right)\right\|_{H^{52-j}} \\
& \quad+\sum_{j=0}^{1}\left\|\partial_{t}^{j}\left(n(t)-n_{+}(t)\right)\right\|_{H^{51-j}} \longrightarrow 0 \quad(t \rightarrow \infty),
\end{aligned}
$$

where

$$
\begin{aligned}
& u_{+}(t)=(\cos \omega t) u_{+0}+\left(\omega^{-1} \sin \omega t\right) u_{+1}, \\
& n_{+}(t)=\left(\cos \omega_{0} t\right) n_{+0}+\left(\omega_{0}^{-1} \sin \omega_{0} t\right) n_{+1} .
\end{aligned}
$$

Remark 1.1. - (1) For $s_{1}>s_{2}>0, L^{p} \nsubseteq \dot{H}^{-s_{2}, p} \nsubseteq \dot{H}^{-s_{1}, p}$ and $\dot{H}^{-s_{1}, p} \cap L^{p} \subseteq \dot{H}^{-s_{2}, p}$. In three space dimensions, $\mathcal{S} \subseteq \dot{H}^{-1}$ but $\mathcal{S} \nsubseteq \dot{H}^{-2}$, where $\mathcal{S}$ is the Schwartz space on $\mathbf{R}^{3}$. For the details of the homogeneous Sobolev space $\dot{H}^{s, p}$, see $[2, \S 6.3$ in Chapter 6]. But note that the definition of $\dot{H}^{s, p}$ in the present paper is slightly different from the one in $[2, \S 6.3]$, where $\dot{H}^{s, p}$ is defined as the set of all tempered distributions $v$ such that $(-\Delta)^{s / 2} v \in L^{p}$. In the general $N$ space dimension case, if we take $v \in \dot{H}^{s, p}\left(\mathbf{R}^{N}\right)$ modulo monomials of degree larger than $[s-N / p]$ in the definition of $[2, \S 6.3]$, then the definition in $[2, \S 6.3]$ is identical to that in the present paper (see [2, Excercise 12 in $\S 6.8]$ ). Here $[s-N / p]$ is the largest integer that is not larger than $s-N / p$ and if $[s-N / p]$ is negative, we take zero as a monomial of order $[s-N / p]$.

(2) $u_{+}(t)$ and $n_{+}(t)$ are the solutions of the free Klein-Gordon equation and the free wave equation with the initial conditions $\left(u_{+}(0), \partial_{t} u_{+}(0)\right)=$ $\left(u_{+0}, u_{+1}\right)$ and $\left(n_{+}(0), \partial_{t} n_{+}(0)\right)=\left(n_{+0}, n_{+1}\right)$, respectively. The relation (1.11) implies that the solutions of (1.1)-(1.3) given by Theorem 1.1 behave like the free solutions as $t \rightarrow \infty$.

(3) In fact, our proof of Theorem 1.1 (or Corollary 1.2 below) will work well, even if we do not assume that $n_{0}$ and $n_{1}$ are real-valued. However, this assumption will be often used in the proof, because it will make the proof slightly simpler. If $n_{0}$ and $n_{1}$ are real-valued, then $n(t, x)$ is a real-valued function. This fact follows immediately from the uniqueness of solutions of (1.1)-(1.3).

(4) In connection with the usual Zakharov system (1.4)-(1.5) for three space dimensions, it is conjectured that if the initial data are large, the solutions of (1.1)-(1.3) may not necessarily exist globally in time. 
(5) In the case of one or two space dimensions, the global existence result for small initial data can be proved more easily than the case of three space dimensions. We do not need the time decay estimates to show the global existence of solutions in the one and two dimensional cases. For the details, see Theorem 2.7 in Section 2.

The following corollary is an immediate consequence of Theorem 1.1.

COROLlaRY 1.2. - Let $0<\varepsilon \leq 10^{-2}$ and let $m$ be a positive integer with $m \geq 52$. Assume that $u_{0} \in H^{m} \cap W^{29,6 /(5+2 \varepsilon)}, u_{1} \in H^{m-1} \cap W^{28,6 /(5+2 \varepsilon)}$, $n_{0} \in H^{m-1} \cap W^{28,220 / 217} \cap \dot{H}^{-1}, n_{1} \in H^{m-2} \cap W^{27,220 / 217} \cap \dot{H}^{-2}$ and $\left(u_{0}, u_{1}, n_{0}, n_{1}\right)$ satisfy (1.6). Then, the solutions $(u, n)$ given by Theorem 1.1 satisfy

$$
\begin{aligned}
& u \in \bigcap_{j=0}^{m} C^{j}\left([0, \infty) ; H^{m-j}\right), \\
& n \in \bigcap_{j=0}^{m-1} C^{j}\left([0, \infty) ; H^{m-1-j}\right) .
\end{aligned}
$$

In addition, if $u_{0}, u_{1}, n_{0}, n_{1} \in \cap_{j=1}^{\infty} H^{j}$, then we have

$$
u(t, x), \quad n(t, x) \in C^{\infty}\left([0, \infty) \times \mathbf{R}^{3}\right)
$$

The unique existence and the regularity of local solutions for (1.1)-(1.3) follows from the standard iteration argument. The crucial part of proofs of Theorem 1.1 and Corollary 1.2 is to establish the a priori estimates of the solutions for (1.1)-(1.3) in order to extend the local solutions globally in time. The global behavior of local solutions for (1.1)-(1.3) can not be controlled directly, since the quadratic nonlinear term in (1.1) does not provide a sufficient decay property for the three dimensional case. Here we use the argument of normal forms of Shatah [12] to transform the quadratic nonlinearity into the cubic one. However, in our problem the transformed cubic nonlinearity is represented in terms of the integral operator with singular kernel. The singularity of the integral kernel makes it difficult to solve (1.1)-(1.3). This is different from the case of the system containing only the Klein-Gordon equations, where the integral kernels of the resulting integral operators are regular (see [12]). Therefore, our main task in the proof of Theorem 1.1 is to evaluate the singularity of the integral kernel of the transformed cubic nonlinearity. This enables us to apply the usual $L^{p}-L^{q}$ estimate to the transformed system, which provides us with 
the sufficient decay properties of solutions to (1.1)-(1.3) for the proof of Theorem 1.1.

Our plan in the present paper is as follows. In Section 2 we prepare several preliminary results needed for the proofs of Theorem 1.1 and Corollary 1.2. We also state the global existence results for the cases of one and two space dimensions at the end of Section 2. In Section 3 we describe the proofs of Theorem 1.1 and Corollary 1.2.

We conclude this section by giving several notations. For $s \in \mathbf{R}$ and $1<p<\infty$, we define $H^{s, p}$ by $H^{s, p}=(1-\Delta)^{-s / 2} L^{p}$. For $p, q \in \mathbf{R}^{N}$, we put $p \cdot q=\sum_{j=1}^{N} p_{j} q_{j}$. For $f \in \mathcal{S}\left(\mathbf{R}^{N}\right)$, we define the Fourier transform $\hat{f}$ and the inverse Fourier transform $\check{f}$ of $f$ by

$$
\begin{aligned}
& \hat{f}(\xi)=\int_{\mathbf{R}^{N}} \mathrm{e}^{-i x \cdot \xi} f(x) d x, \\
& \check{f}(x)=\frac{1}{(2 \pi)^{N}} \int_{\mathbf{R}^{N}} \mathrm{e}^{i x \cdot \xi} f(\xi) d \xi .
\end{aligned}
$$

We also denote the Fourier transform and the inverse Fourier transform of $f$ by $\mathcal{F}[f](\xi)$ and $\mathcal{F}^{-1}[f](x)$, respectively. We put $\langle p\rangle=\left(1+|p|^{2}\right)^{1 / 2}$ for $p \in \mathbf{R}^{N}$. For $s \in \mathbf{R}$, let $[s]$ be the largest integer that is not larger than $s$. For a multi-index $\alpha=\left(\alpha_{1}, \cdots, \alpha_{N}\right)$, we put

$$
\partial_{x}^{\alpha}=\left(\frac{\partial}{\partial x_{1}}\right)^{\alpha_{1}} \cdots\left(\frac{\partial}{\partial x_{N}}\right)^{\alpha_{N}}
$$

For $z \in \mathbf{C}$, we denote the complex conjugate of $z$ by $\bar{z}$. For $u, v \in \mathcal{S}\left(\mathbf{R}^{3}\right)$ and $K(x, y) \in \mathcal{S}^{\prime}\left(\mathbf{R}^{3} \times \mathbf{R}^{3}\right)$, we put

$$
[u, K, v](x)=\iint_{\mathbf{R}^{3} \times \mathbf{R}^{3}} \bar{u}(y) K(x-y, x-z) v(z) d y d z .
$$

In the course of calculations below, the various constants are simply denoted by $C$.

\section{PRELIMINARY RESULTS}

In this section we describe the preliminary results needed for the proofs of Theorem 1.1 and Corollary 1.2.

We first begin with the following lemma concerning the $L^{p}-L^{q}$ estimates of the linear Klein-Gordon and wave equations. 
LEMMA 2.1. - Let $N$ be the arbitrary space dimensions and let $p$ and $q$ be two positive numbers such that $2 \leq p<\infty$ and $\frac{1}{p}+\frac{1}{q}=1$.

(i) Let $0 \leq \theta \leq 1$. We put $\gamma=\frac{N-1+\theta}{2}-\frac{N+1+\theta}{p}$. Then, we have $\left\|\partial_{t}^{j}\left\{\omega^{-1} \exp (i \omega t) v\right\}\right\|_{L^{p}} \leq K(t)\|v\|_{H^{\gamma+j, q}}, t>0, v \in H^{\gamma, q}$, for $j=0,1$, where

$$
K(t)=C \begin{cases}t^{-(N-1-\theta)\left(\frac{1}{2}-\frac{1}{p}\right)}, & 0<t \leq 1 \\ t^{-(N-1+\theta)\left(\frac{1}{2}-\frac{1}{p}\right)}, & t \geq 1\end{cases}
$$

and $C$ depends only on $N, p$ and $\theta$.

(ii) We put $\gamma=\frac{N-1}{2}-\frac{N+1}{p}$. Then, we have

$$
\begin{aligned}
& \left\|\partial_{t}^{j}\left\{\omega_{0}^{-1} \exp \left(i \omega_{0} t\right) v\right\}\right\|_{L^{p}} \\
& \quad \leq C t^{-(N-1)\left(\frac{1}{2}-\frac{1}{p}\right)}\|v\|_{\dot{H}^{\gamma+j, q}}, t>0, v \in \dot{H}^{\gamma, q},
\end{aligned}
$$

for $j=0,1$, where $C$ depends only on $N$ and $p$.

Lemma 2.1 is well known. See, e.g., [8, Lemma 2.1] and [11, part b) of Theorem 0] for Lemma 2.1 (i) and [11, part a) of Theorem 0] for Lemma 2.1 (ii).

We next state the lemma concerning the estimate of the integral kernel, which will be useful in evaluating the cubic nonlinearity of the transformed equations.

LEMMA 2.2. - Assume $0<\eta<\frac{1}{2}$. Let $K_{j}(x, y), j=1,2$, be the tempered distributions on $\mathbf{R}^{3} \times \mathbf{R}^{3}$ such that

$$
\begin{aligned}
& \hat{K}_{1}(p, q)=\frac{|p|^{1+\eta}(p \cdot q)}{2\left\{|p|^{2}|q|^{2}-(p \cdot q)^{2}+|p|^{2}\right\}\langle p\rangle^{2}\langle q\rangle^{8}}, \\
& \hat{K}_{2}(p, q)=-\frac{|p|^{2+\eta}}{2\left\{|p|^{2}|q|^{2}-(p \cdot q)^{2}+|p|^{2}\right\}\langle p\rangle^{2}\langle q\rangle^{7}}
\end{aligned}
$$

for $p, q \in \mathbf{R}^{3}$. Then,

$$
K_{1}(x, y), K_{2}(x, y) \in L^{1}\left(\mathbf{R}^{3} \times \mathbf{R}^{3}\right) .
$$

Proof. - We first show $K_{2}(x, y) \in L^{1}\left(\mathbf{R}^{3} \times \mathbf{R}^{3}\right)$. We easily see that $\hat{K}_{2}(p, q) \in L^{2}\left(\mathbf{R}^{3} \times \mathbf{R}^{3}\right)$ and so $K_{2}(x, y) \in L^{2}\left(\mathbf{R}^{3} \times \mathbf{R}^{3}\right)$. By Schwarz's Vol. $12, n^{\circ} 4-1995$. 
inequality and Parseval's identity, we have

$$
\begin{aligned}
\int_{\mathbf{R}^{3}} & \int_{\mathbf{R}^{3}}\left|K_{2}(x, y)\right| d x d y \\
= & \int_{\mathbf{R}^{3}} \int_{\mathbf{R}^{3}}\left(1+|x|^{2}\right)^{-\frac{3}{4}-\frac{\eta}{8}}\left(1+|y|^{2}\right)^{-1} \\
& \times\left\{\left(1+|x|^{2}\right)^{\frac{3}{4}+\frac{\eta}{8}}\left(1+|y|^{2}\right)\left|K_{2}(x, y)\right|\right\} d x d y \\
\leq & \left(\int_{\mathbf{R}^{3}} \int_{\mathbf{R}^{3}}\left(1+|x|^{2}\right)^{-\frac{3}{2}-\frac{\eta}{4}}\left(1+|y|^{2}\right)^{-2} d x d y\right)^{1 / 2} \\
& \times\left(\int_{\mathbf{R}^{3}} \int_{\mathbf{R}^{3}}\left\{\left(1+|x|^{2}\right)^{\frac{3}{4}+\frac{\eta}{8}}\left(1+|y|^{2}\right)\left|K_{2}(x, y)\right|\right\}^{2} d x d y\right)^{1 / 2} \\
\leq & C\left(\int_{\mathbf{R}^{3}} \int_{\mathbf{R}^{3}}\left|\left(1-\triangle_{p}\right)^{\frac{3}{4}+\frac{\eta}{8}}\left(1-\triangle_{q}\right) \hat{K}_{2}(p, q)\right|^{2} d p d q\right)^{1 / 2},
\end{aligned}
$$

where $\triangle_{p}$ and $\triangle_{q}$ are the Laplace operators with respect to the variables $p$ and $q$, respectively. We have only to show that the integral at the right hand side of (2.1) is finite. We put

$$
f(p, q)=|p|^{2}|q|^{2}-(p \cdot q)^{2}+|p|^{2} .
$$

A simple calculation gives us

$$
\begin{aligned}
\left(1-\triangle_{q}\right) \hat{K}_{2}= & \hat{K}_{2}-\frac{2|p|^{4+\eta}}{f^{2}\langle p\rangle^{2}\langle q\rangle^{7}}+\frac{4\left\{|p|^{2}|q|^{2}-(p \cdot q)^{2}\right\}|p|^{4+\eta}}{f^{3}\langle p\rangle^{2}\langle q\rangle^{7}} \\
& -\frac{21|p|^{2+\eta}}{2 f\langle p\rangle^{2}\langle q\rangle^{9}}+\frac{63|p|^{2+\eta}|q|^{2}}{2 f\langle p\rangle^{2}\langle q\rangle^{11}} \\
& +\frac{14\left(|p|^{2}|q|^{2}-(p \cdot q)^{2}\right)|p|^{2+\eta}}{f^{2}\langle p\rangle^{2}\langle q\rangle^{9}} .
\end{aligned}
$$

We note that for some positive constant $M$,

$$
\begin{aligned}
& \frac{1}{M}\left\|(1-\triangle)^{\frac{3}{4}+\frac{\eta}{8}} v\right\|_{L^{2}} \\
& \leq\|v\|_{L^{2}}+\sum_{j=1}^{3}\left\{\int _ { \mathbf { R } ^ { 3 } } \left(|h|^{-\frac{1}{2}-\frac{\eta}{4}}|| \frac{\partial}{\partial x_{j}} v(\cdot+h)\right.\right. \\
& \left.\left.-\frac{\partial}{\partial x_{j}} v(\cdot) \|_{L^{2}}\right)^{2} \frac{d h}{|h|^{3}}\right\}^{1 / 2} \\
& \leq M\left\|(1-\triangle)^{\frac{3}{4}+\frac{\eta}{8}} v\right\|_{L^{2}}
\end{aligned}
$$


(see, e.g., [2, Exercise 7 in Section 6.8]). Therefore, it is sufficient to prove

$$
\begin{aligned}
& \left\|\left(1-\triangle_{q}\right) \hat{K}_{2}(\cdot, q)\right\|_{L^{2}} \\
& +\sum_{j=1}^{3}\left\{\int _ { \mathbf { R } ^ { 3 } } \left(|h|^{-\frac{1}{2}-\frac{\eta}{4}}|| \frac{\partial}{\partial p_{j}}\left(1-\triangle_{q}\right) \hat{K}_{2}(\cdot+h, q)\right.\right. \\
& \left.\quad-\frac{\partial}{\partial p_{j}}\left(1-\triangle_{q}\right) \hat{K}_{2}(\cdot, q) \|_{L^{2}}\right)^{2} \\
& \left.\times \frac{d h}{|h|^{3}}\right\}^{1 / 2} \in L^{2}\left(\mathbf{R}_{q}^{3}\right) .
\end{aligned}
$$

By (2.2) we have

$$
\begin{aligned}
& \frac{\partial}{\partial p_{j}}\left(1-\triangle_{q}\right) \hat{K}_{2}=\frac{\partial}{\partial p_{j}} \hat{K}_{2}-\frac{2(4+\eta)|p|^{2+\eta} p_{j}}{f^{2}\langle p\rangle^{2}\langle q\rangle^{7}} \\
& \quad+\frac{8\left\{p_{j}|q|^{2}-q_{j}(p \cdot q)+p_{j}\right\}|p|^{4+\eta}}{f^{3}\langle p\rangle^{2}\langle q\rangle^{7}}+\frac{4|p|^{4+\eta} p_{j}}{f^{2}\langle p\rangle^{4}\langle q\rangle^{7}} \\
& \quad+\frac{8\left\{p_{j}|q|^{2}-q_{j}(p \cdot q)\right\}|p|^{4+\eta}+4(4+\eta)\left\{|p|^{2}|q|^{2}-(p \cdot q)^{2}\right\}|p|^{2+\eta} p_{j}}{f^{3}\langle p\rangle^{2}\langle q\rangle^{7}} \\
& \quad-\frac{24\left\{p_{j}|q|^{2}-q_{j}(p \cdot q)+p_{j}\right\}\left\{|p|^{2}|q|^{2}-(p \cdot q)^{2}\right\}|p|^{4+\eta}}{f^{4}\langle p\rangle^{2}\langle q\rangle^{7}} \\
& \quad-\frac{4\left\{|p|^{2}|q|^{2}-(p \cdot q)^{2}\right\}|p|^{4+\eta} p_{j}}{f^{3}\langle p\rangle^{4}\langle q\rangle^{7}}-\frac{21(2+\eta)|p|^{\eta} p_{j}}{2 f\langle p\rangle^{2}\langle q\rangle^{9}} \\
& \quad+\frac{21\left\{p_{j}|q|^{2}-q_{j}(p \cdot q)+p_{j}\right\}|p|^{2+\eta}}{f^{2}\langle p\rangle^{2}\langle q\rangle^{9}}+\frac{21|p|^{2+\eta} p_{j}}{f\langle p\rangle^{4}\langle q\rangle^{9}} \\
& +\frac{63(2+\eta)|p|^{\eta} p_{j}|q|^{2}}{2 f\langle p\rangle^{2}\langle q\rangle^{11}}-\frac{63\left\{p_{j}|q|^{2}-q_{j}(p \cdot q)+p_{j}\right\}|p|^{2+\eta}|q|^{2}}{f^{2}\langle p\rangle^{2}\langle q\rangle^{11}} \\
& \quad-\frac{63|p|^{2+\eta} p_{j}|q|^{2}}{f\langle p\rangle^{4}\langle q\rangle^{11}}+\frac{28\left(p_{j}|q|^{2}-q_{j}(p \cdot q)\right)|p|^{2+\eta}}{f^{2}\langle p\rangle^{2}\langle q\rangle^{9}} \\
& +\frac{14(2+\eta)\left(|p|^{2}|q|^{2}-(p \cdot q)^{2}\right)|p|^{\eta} p_{j}}{f^{2}\langle p\rangle^{2}\langle q\rangle^{9}} \\
& \quad-\frac{56\left(|p|^{2}|q|^{2}-(p \cdot q)^{2}\right)\left(p_{j}|q|^{2}-q_{j}(p \cdot q)+p_{j}\right)|p|^{2+\eta}}{f^{3}\langle p\rangle^{2}\langle q\rangle^{9}} \\
& \quad-\frac{28\left(|p|^{2}|q|^{2}-(p \cdot q)^{2}\right)|p|^{2+\eta} p_{j}}{f^{2}\langle p\rangle^{4}\langle q\rangle^{9}},
\end{aligned}
$$




$$
\begin{aligned}
\frac{\partial}{\partial p_{j}} \hat{K}_{2}= & -\frac{(2+\eta)|p|^{\eta} p_{j}}{2 f\langle p\rangle^{2}\langle q\rangle^{7}} \\
& +\frac{\left\{p_{j}|q|^{2}-q_{j}(p \cdot q)+p_{j}\right\}|p|^{2+\eta}}{f^{2}\langle p\rangle^{2}\langle q\rangle^{7}}+\frac{|p|^{2+\eta} p_{j}}{f\langle p\rangle^{4}\langle q\rangle^{7}}
\end{aligned}
$$

for $1 \leq j \leq 3$. We concentrate upon the estimate of the sixth term at the right hand side of (2.5), since the rest terms of (2.2), (2.5) and (2.6) are easier to treat.

By $I_{j}(p, q)$ we denote the sixth term at the right hand side of (2.5) for $1 \leq j \leq 3$. We show that

$$
\left\{\int_{\mathbf{R}^{3}} \int_{\mathbf{R}^{3}}\left|I_{j}(p+h, q)-I_{j}(p, q)\right|^{2} d p|h|^{-4-\frac{\eta}{2}} d h\right\}^{\frac{1}{2}} \in L^{2}\left(\mathbf{R}_{q}^{3}\right)
$$

for $1 \leq j \leq 3$. We divide the integral region of (2.7) into four parts:

$$
\begin{aligned}
& D_{1}=\left\{(p, h) ;|p|<2,|h|<1,|h|<\frac{1}{2}|p|\right\}, \\
& D_{2}=\left\{(p, h) ;|p|<2,|h|<1,|h|>\frac{1}{2}|p|\right\}, \\
& D_{3}=\{(p, h) ;|p|>2,|h|<1\}, \\
& D_{4}=\{(p, h) ;|h|>1\} .
\end{aligned}
$$

We show (2.7) for each integral region $D_{j}$.

(Estimate on $\left.D_{1}\right)$. Since $\langle p\rangle^{-2} \in C^{\infty}\left(\mathbf{R}^{3}\right)$, we may evaluate $\tilde{I}_{j}(p, q)=$ $\langle p\rangle^{2} I_{j}(p, q)$ instead of $I_{j}(p, q)$ in this case. We put

$$
F_{j}(p, q)=24\left\{p_{j}|q|^{2}-q_{j}(p \cdot q)+p_{j}\right\}\left\{|p|^{2}|q|^{2}-(p \cdot q)^{2}\right\}|p|^{4+\eta} .
$$

Then, we have

$$
\begin{aligned}
\tilde{I}_{j}(p+h, q)-\tilde{I}_{j}(p, q) & \\
= & \frac{F_{j}(p+h, q)}{\langle q\rangle^{7}}\left(\frac{1}{f(p+h, q)^{4}}-\frac{1}{f(p, q)^{4}}\right) \\
& +\frac{F_{j}(p+h, q)-F_{j}(p, q)}{f(p, q)^{4}\langle q\rangle^{7}} \\
= & A_{j}+B_{j} .
\end{aligned}
$$

We first evaluate $A_{j}$. Since $|h|<\frac{1}{2}|p|$, we have

$$
\left|F_{j}(p+h, q)\right| \leq C\langle q\rangle^{2}|q|^{2}|p|^{7+\eta} .
$$


On the other hand, noting $f(p, q) \geq 0$, we have

$$
\begin{aligned}
\left|\frac{1}{f(p+h, q)^{4}}-\frac{1}{f(p, q)^{4}}\right| & \\
= & \left|\left(f(p+h, q)^{\frac{1}{2}+\frac{\eta}{2}}\right)^{-8 /(1+\eta)}-\left(f(p, q)^{\frac{1}{2}+\frac{\eta}{2}}\right)^{-8 /(1+\eta)}\right| \\
= & \mid-\frac{8}{1+\eta} \int_{0}^{1}\left(\theta f(p+h, q)^{\frac{1+\eta}{2}}\right. \\
& \left.+(1-\theta) f(p, q)^{\frac{1+\eta}{2}}\right)^{-(9+\eta) /(1+\eta)} d \theta \\
& \times\left(f(p+h, q)^{\frac{1+\eta}{2}}-f(p, q)^{\frac{1+\eta}{2}}\right) \mid \\
\leq & C\left\{\int_{1 / 2}^{1} \theta^{\frac{-(9+\eta)}{1+\eta}} d \theta f(p+h, q)^{-\frac{9+\eta}{2}}\right. \\
& \left.\quad+\int_{0}^{1 / 2}(1-\theta)^{-\frac{9+\eta}{1+\eta}} d \theta f(p, q)^{-\frac{9+\eta}{2}}\right\} \\
& \times\left|f(p+h, q)^{\frac{1+\eta}{2}}-f(p, q)^{\frac{1+\eta}{2}}\right| \\
\leq & C\left\{f(p+h, q)^{-\frac{9+\eta}{2}}+f(p, q)^{-\frac{9+\eta}{2}}\right\} \\
& \times|f(p+h, q)-f(p)|^{(1+\eta) / 2} .
\end{aligned}
$$

At the last inequality we have used the fact that $\left|a^{\frac{1+\eta}{2}}-b^{\frac{1+\eta}{2}}\right| \leq$ $C|a-b|^{(1+\eta) / 2}$ for $a, b \geq 0$. Moreover, we easily see that for $|h|<\frac{1}{2}|p|$,

$$
\begin{aligned}
& f(p+h, q)^{-\frac{9+\eta}{2}} \leq C|p+h|^{-9-\eta} \\
& \quad \leq\left. C|| p|-| h\right|^{-9-\eta} \leq C|p|^{-9-\eta} \\
& |f(p+h, q)-f(p, q)|^{\frac{1+\eta}{2}} \\
& \quad \leq C\langle q\rangle^{1+\eta}|p|^{\frac{1+\eta}{2}}|h|^{\frac{1+\eta}{2}} .
\end{aligned}
$$

Accordingly, (2.10)-(2.12) yield

$$
\begin{aligned}
& \left|\frac{1}{f(p+h, q)^{4}}-\frac{1}{f(p, q)^{4}}\right| \\
& \quad \leq C\left(|p|^{-9-\eta}+|p|^{-9-\eta}\right)\langle q\rangle^{1+\eta}|p|^{\frac{1+\eta}{2}}|h|^{\frac{1+\eta}{2}} \\
& \quad \leq C\langle q\rangle^{1+\eta}|p|^{-\frac{17+\eta}{2}}|h|^{\frac{1+\eta}{2}}
\end{aligned}
$$


for $|h|<\frac{1}{2}|p|$. By (2.9) and (2.13) we obtain

$$
\begin{aligned}
\left|A_{j}\right| & \leq C\langle q\rangle^{-7}\langle q\rangle^{2}|q|^{2}|p|^{7+\eta}\langle q\rangle^{1+\eta}|p|^{-\frac{17+\eta}{2}}|h|^{\frac{1+\eta}{2}} \\
& \leq C\langle q\rangle^{-2+\eta}|p|^{-\frac{3}{2}+\frac{\eta}{2}}|h|^{\frac{1+\eta}{2}}
\end{aligned}
$$

for $|h|<\frac{1}{2}|p|$.

We next evaluate $B_{j}$. A direct calculation yields

$$
\begin{aligned}
& \left|F_{j}(p+h, q)-F_{j}(p)\right| \\
& \quad \leq \int_{0}^{1}\left|\left(\nabla_{p} F_{j}\right)(p+(1-\theta) h, q) \cdot h\right| d \theta \\
& \quad \leq C\langle q\rangle^{2}|q|^{2}|p|^{6+h}|h| \\
& \quad \leq C\langle q\rangle^{2}|q|^{2}|p|^{\frac{13+\eta}{2}}|h|^{\frac{1+\eta}{2}}\left(\frac{|h|}{|p|}\right)^{\frac{1}{2}-\frac{\eta}{2}} \\
& \quad \leq C\langle q\rangle^{2}|q|^{2}|p|^{\frac{13+\eta}{2}}|h|^{\frac{1+\eta}{2}}
\end{aligned}
$$

for $|h| \leq \frac{1}{2}|p|$. By (2.15) we obtain

$$
\begin{aligned}
\left|B_{j}\right| & \leq C|p|^{-8}\langle q\rangle^{-7}\langle q\rangle^{2}|q|^{2}|p|^{\frac{13+\eta}{2}}|h|^{\frac{1+\eta}{2}} \\
& \leq C\langle q\rangle^{-3}|p|^{-\frac{3}{2}+\frac{\eta}{2}}|h|^{\frac{1+\eta}{2}}
\end{aligned}
$$

for $|h| \leq \frac{1}{2}|p|$.

Therefore, we obtain by (2.8), (2.14) and (2.16)

$$
\begin{aligned}
& \left|\tilde{I}_{j}(p+h, q)-\tilde{I}_{j}(p, q)\right| \\
& \quad \leq C\left(\langle q\rangle^{-2+\eta}|p|^{-\frac{3}{2}+\frac{\eta}{2}}|h|^{\frac{1+\eta}{2}}+\langle q\rangle^{-3}|p|^{-\frac{3}{2}+\frac{\eta}{2}}|h|^{\frac{1+\eta}{2}}\right) \\
& \quad \leq C\langle q\rangle^{-2+\eta}|p|^{-\frac{3}{2}+\frac{\eta}{2}}|h|^{\frac{1+\eta}{2}}
\end{aligned}
$$

for $(p, h) \in D_{1}$. Inequality (2.17) gives us

$$
\begin{aligned}
\text { (2.18) }\left\{\iint_{D_{1}}\left|\tilde{I}_{j}(p+h, q)-\tilde{I}_{j}(p, q)\right|^{2} d p|h|^{-4-\frac{\eta}{2}} d h\right\}^{1 / 2} \\
\leq C\left\{\left(\int_{|p|<2}|p|^{-3+\eta} d p\right)\left(\int_{|h|<1}|h|^{-3+\frac{\eta}{2}} d h\right)\right\}^{1 / 2}\langle q\rangle^{-2+\eta} .
\end{aligned}
$$


Since $0<\eta<1 / 2$, (2.18) shows that the right hand side of (2.18) belongs to $L^{2}\left(\mathbf{R}_{q}^{3}\right)$. Since $\langle p\rangle^{-2} \in C^{\infty}\left(\mathbf{R}^{3}\right)$ and $I_{j}(p, q)=\langle p\rangle^{-2} \tilde{I}_{j}(p, q)$, we conclude that

$$
\left\{\iint_{D_{1}}\left|I_{j}(p+h, q)-I_{j}(p, q)\right|^{2} d p|h|^{-4-\frac{\eta}{2}} d h\right\}^{1 / 2} \in L^{2}\left(\mathbf{R}_{q}^{3}\right)
$$

for $1 \leq j \leq 3$.

(Estimate on $\left.D_{2}\right)$. We may evaluate $\tilde{I}_{j}(p, q)=\langle p\rangle^{2} I_{j}(p, q)$ instead of $I_{j}(p, q)$ for the same reason as the case of $D_{1}$.

We have

$$
\begin{aligned}
& \iint_{D_{2}}\left|\tilde{I}_{j}(p+h, q)-\tilde{I}_{j}(p, q)\right|^{2}|h|^{-4-\frac{\eta}{2}} d p d h \\
& \quad \leq C \int_{|h|<1}|h|^{-4-\frac{\eta}{2}} d h \int_{|p|<2|h|}\left(\left|\tilde{I}_{j}(p+h, q)\right|^{2}+\left|\tilde{I}_{j}(p, q)\right|^{2}\right) d p
\end{aligned}
$$

By the change of variables $p^{\prime}=p+h$, we obtain

$$
\begin{aligned}
& \int_{|h|<1}|h|^{-4-\frac{\eta}{2}} d h \int_{|p|<2|h|}\left|\tilde{I}_{j}(p+h, q)\right|^{2} d p \\
& \leq \int_{|h|<1}|h|^{-4-\frac{\eta}{2}} d h \int_{\left|p^{\prime}\right|<3|h|}\left|\tilde{I}_{j}\left(p^{\prime}, q\right)\right|^{2} d p^{\prime} \\
& \leq C\langle q\rangle^{-6} \int_{0}^{1}|h|^{-2-\frac{\eta}{2}} d|h| \int_{0}^{3|h|}\left|p^{\prime}\right|^{2 \eta} d\left|p^{\prime}\right| \\
& \leq C\langle q\rangle^{-6} \int_{0}^{1}|h|^{-1+\frac{3}{2} \eta} d|h| \\
& \leq C\langle q\rangle^{-6} .
\end{aligned}
$$

At the second inequality of (2.21) we have used the following relation:

$$
\left|\tilde{I}_{j}(p, q)\right| \leq C\langle q\rangle^{-3}|p|^{-1+\eta}
$$

We can similarly obtain

$$
\int_{|h|<1}|h|^{-4-\frac{\eta}{2}} d h \int_{|p|<2|h|}\left|\tilde{I}_{j}(p, q)\right|^{2} d p \leq C\langle q\rangle^{-6}
$$

Therefore, (2.20), (2.21) and (2.23) yield

$$
\left\{\iint_{D_{2}}\left|\tilde{I}_{j}(p+h, q)-\tilde{I}_{j}(p, q)\right|^{2}|h|^{-4-\frac{\eta}{2}} d p d h\right\}^{1 / 2} \in L^{2}\left(\mathbf{R}_{q}^{3}\right)
$$


for $1 \leq j \leq 3$. Since $\langle p\rangle^{-2} \in C^{\infty}\left(\mathbf{R}^{3}\right)$ and $I_{j}(p, q)=\langle p\rangle^{-2} \tilde{I}_{j}(p, q)$, we conclude that

$$
\left\{\iint_{D_{2}}\left|I_{j}(p+h, q)-I_{j}(p, q)\right|^{2}|h|^{-4-\frac{\eta}{2}} d p d h\right\}^{1 / 2} \in L^{2}\left(\mathbf{R}_{q}^{3}\right)
$$

for $1 \leq j \leq 3$.

(Estimate on $D_{3}$ ). Since $I_{j}(p, q), 1 \leq j \leq 3$ have no singularity for $|p|>2$, it easily follows that for $1 \leq \bar{j} \leq \overline{3}$,

$$
\left\{\iint_{D_{3}}\left|I_{j}(p+h, q)-I_{j}(p, q)\right|^{2}|h|^{-4-\frac{\eta}{2}} d p d h\right\}^{1 / 2} \in L^{2}\left(\mathbf{R}_{q}^{3}\right) .
$$

We omit the proof of (2.26).

(Estimate on $\left.D_{4}\right)$. Since $I_{j}(p, q)=\langle p\rangle^{-2} \tilde{I}_{j}(p, q)$, by (2.22) we have

$$
\left|I_{j}(p, q)\right| \leq C\langle q\rangle^{-3}\langle p\rangle^{-2}|p|^{-1+\eta} .
$$

By the change of variables $p^{\prime}=p+h$ and (2.27), we obtain

$$
\begin{aligned}
& \iint_{D_{4}}\left|I_{j}(p+h, q)-I_{j}(p, q)\right|^{2}|h|^{-4-\frac{\eta}{2}} d p d h \\
& \leq C \int_{|h|>1} \int_{\mathbf{R}^{3}}\left\{\left|I_{j}(p+h, q)\right|^{2}+\left|I_{j}(p, q)\right|^{2}\right\} \\
& \times|h|^{-4-\frac{\eta}{2}} d p d h \\
& \leq C \int_{|h|>1}|h|^{-4-\frac{\eta}{2}} \int_{\mathbf{R}^{3}}\left|I_{j}(p+h, q)\right|^{2} d p d h \\
&+C \int_{|h|>1}|h|^{-4-\frac{\eta}{2}} \int_{\mathbf{R}^{3}}\left|I_{j}(p, q)\right|^{2} d p d h \\
&= 2 C\left(\int_{|h|>1}|h|^{-4-\frac{\eta}{2}} d h\right)\left(\int_{\mathbf{R}^{3}}\left|I_{j}(p, q)\right|^{2} d p\right) \\
& \leq C\left(\int_{1}^{\infty}|h|^{-2-\frac{\eta}{2}} d|h|\right)\left(\int_{0}^{\infty}\langle p\rangle^{-4+2 \eta} d|p|\right)\langle q\rangle^{-6} \\
& \leq C\langle q\rangle^{-6} .
\end{aligned}
$$

At the last inequality we have used the assumption that $0<\eta<1 / 2$. Accordingly, (2.28) gives us

$$
\left\{\iint_{D_{4}}\left|I_{j}(p+h, q)-I_{j}(p, q)\right|^{2}|h|^{-4-\frac{\eta}{2}} d p d h\right\}^{1 / 2} \in L^{2}\left(\mathbf{R}_{q}^{3}\right)
$$


for $1 \leq j \leq 3$.

Thus, (2.19), (2.25), (2.26) and (2.29) show (2.7). Since the other terms of (2.2), (2.5) and (2.6) can be evaluated more easily, we obtain Lemma 2.2 for $K_{2}(x, y)$.

In the same way as above we can prove Lemma 2.2 for $K_{1}(x, y)$.

(Q.E.D.)

Remark 2.1. - We note that $\hat{K}_{j} \notin H^{s}\left(\mathbf{R}^{6}\right), j=1,2$ for $s>3$, because the singularity of $\hat{K}_{j}(p, q)$ near $p=0$ is too strong. Therefore, for example, Lemma 6.1.5 in Chapter 6 of [2], which is well known, can not be applied to $K_{1}$ and $K_{2}$. The Fourier multiplier theorem of the Mihlin type can not be applied to $K_{1}$ and $K_{2}$, either (see, e.g., [2, Theorem 6.1.6 in Chapter 6]). This is because the set of singular points of $\hat{K}_{1}$ and $\hat{K}_{2}$ is not one point but the region $\left\{(p, q) \in \mathbf{R}^{3} \times \mathbf{R}^{3} ; p=0\right\}$.

The following corollary follows immediately from the proof of Lemma 2.2.

Corollary 2.3. - Assume $0<\eta<1 / 2$. Let $J_{j}(p, q), j=1,2$ be the tempered distributions on $\mathbf{R}^{3} \times \mathbf{R}^{3}$ such that

$$
\begin{aligned}
& \hat{J}_{1}(p, q)=\frac{(p \cdot q-1)|p+q|^{2+\eta}}{2\left\{|p|^{2}|q|^{2}-(p \cdot q)^{2}+|p+q|^{2}\right\}\langle p+q\rangle^{2}\langle p-q\rangle^{8}\langle p\rangle\langle q\rangle}, \\
& \hat{J}_{2}(p, q)=-\frac{|p+q|^{2+\eta}}{2\left\{|p|^{2}|q|^{2}-(p \cdot q)^{2}+|p+q|^{2}\right\}\langle p+q\rangle^{2}\langle p-q\rangle^{8}}
\end{aligned}
$$

for $p, q \in \mathbf{R}^{3}$. Then,

$$
J_{1}(x, y), J_{2}(x, y) \in L^{1}\left(\mathbf{R}^{3} \times \mathbf{R}^{3}\right) .
$$

Proof. - We use the change of variables $r=\frac{1}{\sqrt{2}}(p+q)$ and $s=\frac{1}{\sqrt{2}}(p-q)$. Then, we have

$$
\hat{J}_{2}(r, s)=\frac{2^{-1-\eta / 2}|r|^{2+\eta}}{\left(|r|^{2}|s|^{2}-(r \cdot s)^{2}+|r|^{2}\right)\left\langle\frac{1}{\sqrt{2}} r\right\rangle^{2}\left\langle\frac{1}{\sqrt{2}} s\right\rangle^{8}}
$$

Since the above change of variables is a unitary transform from $\mathbf{R}^{6}$ to $\mathbf{R}^{6}$ and $\left\langle\frac{1}{\sqrt{2}} s\right\rangle^{-1} \in W^{4, \infty}\left(\mathbf{R}^{3}\right)$, the proof of Lemma 2.2 implies that $J_{2}(x, y) \in L^{1}\left(\mathbf{R}^{3} \times \mathbf{R}^{3}\right)$.

Vol. $12, n^{\circ} 4-1995$. 
Noting $(p \cdot q-1) /(\langle p\rangle\langle q\rangle) \in W^{4, \infty}\left(\mathbf{R}^{6}\right)$, we can show $J_{1}(x, y) \in$ $L^{1}\left(\mathbf{R}^{6}\right)$ in the same way as $J_{2}(x, y)$.

(Q.E.D.)

We next state an elementary lemma concerning the properties of the integral operators appearing in the normal form of (1.1)-(1.2) (see, e.g., (3.14) and (3.15)).

Lemma 2.4. - (i) Assume $K(x, y) \in L^{1}\left(\mathbf{R}^{3} \times \mathbf{R}^{3}\right)$. Let $p, q$ and $r$ be three numbers such that $1 \leq p, q, r \leq \infty$ and $\frac{1}{r}=\frac{1}{p}+\frac{1}{q}$. Then,

$$
\begin{aligned}
& \left\|\iint_{\mathbf{R}^{3} \times \mathbf{R}^{3}} K(x-y, x-z) f(y) g(z) d y d z\right\|_{L^{r}} \\
& \quad \leq\|K\|_{L^{1}\left(\mathbf{R}^{6}\right)}\|f\|_{L^{p}}\|g\|_{L^{q}}, f \in L^{p}, g \in L^{q} .
\end{aligned}
$$

(ii) Let $K(x, y)$ be a tempered distribution on $\mathbf{R}^{3} \times \mathbf{R}^{3}$ and let $\alpha>0$. We put $K_{\alpha}(x, y)=\mathcal{F}^{-1}\left[|p+q|^{\alpha} \hat{K}(p, q)\right]$. Then,

$$
\begin{aligned}
(- & \triangle)^{\alpha / 2} \iint_{\mathbf{R}^{3} \times \mathbf{R}^{3}} K(x-y, x-z) f(y) g(z) d y d z \\
& =\iint_{\mathbf{R}^{3} \times \mathbf{R}^{3}} K_{\alpha}(x-y, x-z) f(y) g(z) d y d z, f, g \in \mathcal{S} .
\end{aligned}
$$

Proof. - Part (i) follows immediately from Hölder's inequality.

We show (ii). By the change of variables $\tilde{y}=x-y$ and $\tilde{z}=x-z$, we have

$$
\begin{aligned}
& \iint_{\mathbf{R}^{3} \times \mathbf{R}^{3}} K(x-y, x-z) f(y) g(z) d y d z \\
&= \iint K(x-y, x-z) \\
& \times(2 \pi)^{-6}\left(\int e^{i y \cdot p} \hat{f}(p) d p\right)\left(\int e^{i z \cdot q} \hat{g}(q) d q\right) d y d z \\
&=(2 \pi)^{-6} \iint\left[\iint e^{i y \cdot p+i z \cdot q} K(x-y, x-z) d y d z\right] \\
& \times \hat{f}(p) \hat{g}(q) d p d q \\
&=(2 \pi)^{-6} \iint e^{i \boldsymbol{x} \cdot(p+q)}\left[\iint e^{-i(\tilde{y} \cdot p+\tilde{z} \cdot q)} K(\tilde{y}, \tilde{z}) d \tilde{y} d \tilde{z}\right] \\
& \times \hat{f}(p) \hat{g}(q) d p d q \\
&=(2 \pi)^{-6} \iint e^{i x \cdot(p+q)} \hat{K}(p, q) \hat{f}(p) \hat{g}(q) d p d q .
\end{aligned}
$$


On the other hand, we note that

$$
\begin{aligned}
(-\triangle)^{\alpha / 2} e^{i x \cdot(p+q)} & =\mathcal{F}^{-1}\left[|\xi|^{\alpha} \delta(\xi-(p+q))\right] \\
& =\int e^{i x \cdot \xi}|\xi|^{\alpha} \delta(\xi-(p+q)) d \xi \\
& =e^{i x \cdot(p+q)}|p+q|^{\alpha} .
\end{aligned}
$$

The above two identities yield

$$
\begin{aligned}
& (-\triangle)^{\alpha / 2} \iint_{\mathbf{R}^{3} \times \mathbf{R}^{3}} K(x-y, x-z) f(y) g(z) d y d z \\
& =(2 \pi)^{-6} \iint_{\mathbf{R}^{3} \times \mathbf{R}^{3}} e^{i x \cdot(p+q)}|p+q|^{\alpha} \hat{K}(p, q) \hat{f}(p) \hat{g}(q) d p d q \\
& =(2 \pi)^{-6} \iint_{\mathbf{R}^{3} \times \mathbf{R}^{3}} e^{i x \cdot(p+q)} \hat{K}_{\alpha}(p, q) \hat{f}(p) \hat{g}(q) d p d q
\end{aligned}
$$

which implies that (ii) holds.

(Q.E.D.)

We now describe the lemma concerning the $L^{2}$ estimate of quadratic term, which will be useful for the evaluation of the nonlinear term of (1.1).

Lemma 2.5. - Assume that the spatial dimensions are three. Let $0<\varepsilon<10^{-2}$. Then,

$$
\begin{aligned}
& \|v w\|_{H^{51}} \leq C\left\{\|v\|_{H^{51}}\|w\|_{W^{25,6 /(1-2 \varepsilon)}}\right. \\
& \left.\quad+\|v\|_{W^{25,220 / 3}}\|w\|_{W^{26,6 /(1-2 \varepsilon)}}^{1-a}\|w\|_{H^{52}}^{a}\right\}
\end{aligned}
$$

where $C$ depends only on $\varepsilon$, and

$$
a=(5289-220 \varepsilon) /(5500-220 \varepsilon) .
$$

Furthermore, the following relation holds

$$
\frac{107}{110}+(1-a)(1+\varepsilon) \geq 1+\varepsilon
$$

Proof. - We first evaluate the $L^{2}$ norm of the 51st derivatives of $v w$. We have

$$
\begin{aligned}
& \sum_{|\alpha|=51}\left\|\partial_{x}^{\alpha}(v w)\right\|_{L^{2}} \\
& \leq C \sum_{\left|\alpha_{1}\right|+\left|\alpha_{2}\right|=51}\left\|\partial_{x}^{\alpha_{1}} v \partial_{x}^{\alpha_{2}} w\right\|_{L^{2}}
\end{aligned}
$$

Vol. $12, \mathbf{n}^{\circ}$ 4-1995. 


$$
\begin{aligned}
= & C \sum_{\substack{\left|\alpha_{1}\right|+\left|\alpha_{2}\right|=51 \\
26 \leq\left|\alpha_{1}\right| \leq 50}}\left\|\partial_{x}^{\alpha_{1}} v \partial_{x}^{\alpha_{2}} w\right\|_{L^{2}} \\
& +C \sum_{\substack{\left|\alpha_{1}\right|+\left|\alpha_{2}\right|=51 \\
\left|\alpha_{1}\right|=51}}\left\|\partial_{x}^{\alpha_{1}} v \partial_{x}^{\alpha_{2}} w\right\|_{L^{2}} \\
& +C \sum_{\substack{\left|\alpha_{1}\right|+\left|\alpha_{2}\right|=51 \\
\left|\alpha_{1}\right| \leq 25}}\left\|\partial_{x}^{\alpha_{1}} v \partial_{x}^{\alpha_{2}} w\right\|_{L^{2}} \\
= & I_{1}+I_{2}+I_{3} .
\end{aligned}
$$

We note that if $26 \leq\left|\alpha_{1}\right| \leq 50$, then $\left|\alpha_{2}\right| \leq 25$. Therefore, we have by the Sobolev imbedding theorem

$$
\begin{aligned}
I_{1} & \leq C \sum_{\substack{\left|\alpha_{1}\right|+\left|\alpha_{2}\right|=51 \\
\left|\alpha_{1}\right| \leq 50,\left|\alpha_{2}\right| \leq 25}}\left\|\partial_{x}^{\alpha_{1}} v\right\|_{L^{3 /(1+\varepsilon)}}\left\|\partial_{x}^{\alpha_{2}} w\right\|_{L^{6 /(1-2 \varepsilon)}} \\
& \leq C\|v\|_{H^{51}}\|w\|_{W^{25,6 /(1-2 \varepsilon)}}
\end{aligned}
$$

We next note that if $\left|\alpha_{1}\right|=51$, then $\left|\alpha_{2}\right|=0$. Hence, we have by the Sobolev imbedding theorem

$$
\begin{aligned}
I_{2} & \leq C \sum_{\left|\alpha_{1}\right|=51}\left\|\partial_{x}^{\alpha_{1}} v\right\|_{L^{2}}\|w\|_{L^{\infty}} \\
& \leq C\|v\|_{H^{51}}\|w\|_{W^{1,6 /(1-2 \varepsilon)}} .
\end{aligned}
$$

Moreover, we have by Hölder's inequality

$$
\begin{aligned}
I_{3} & \leq C \sum_{\substack{\left|\alpha_{1}\right|+\left|\alpha_{2}\right|=51 \\
\left|\alpha_{1}\right| \leq 25}}\left\|\partial_{x}^{\alpha_{1}} v\right\|_{L^{220 / 3}}\left\|\partial_{x}^{\alpha_{2}} w\right\|_{L^{220 / 107}} \\
& \leq C\left\|\left.v\right|_{W^{25,220 / 3}} \sum_{26 \leq\left|\alpha_{2}\right| \leq 51}\right\| \partial_{x}^{\alpha_{2}} w \|_{L^{220 / 107}}
\end{aligned}
$$

Let $\beta$ be a multi-index such that $|\beta|=\left|\alpha_{2}\right|-25$ and $\beta_{i} \leq \alpha_{2 i}, 1 \leq i \leq 3$, where $\beta=\left(\beta_{1}, \beta_{2}, \beta_{3}\right)$ and $\alpha_{2}=\left(\alpha_{21}, \alpha_{22}, \alpha_{23}\right)$. The application of the Gagliardo-Nirenberg inequality yields

$$
\begin{aligned}
\left\|\partial_{x}^{\alpha_{2}} w\right\|_{L^{220 / 107}} & =\left\|\partial_{x}^{\alpha_{2}-\beta}\left(\partial_{x}^{\beta} w\right)\right\|_{L^{220 / 107}} \\
& \leq C\left\|\partial_{x}^{\beta} w\right\|_{L^{6 /(1-2 \varepsilon)}}^{1-a}\left\|\partial_{x}^{\beta} w\right\|_{H^{26}}^{a}
\end{aligned}
$$


where

$$
a=(5289-220 \varepsilon) /(5500-220 \varepsilon)
$$

(for the Gagliardo-Nirenberg inequality, see, e.g., [4]). For the proof of (2.36), we need the following relation

$$
\frac{\left|\alpha_{2}\right|-|\beta|}{\left|\alpha_{2}\right|-|\beta|+1} \leq a
$$

which follows from the facts that $\left|\alpha_{2}\right|-|\beta|=25$ and that $0<\varepsilon<10^{-2}$. Since $|\beta|=\left|\alpha_{2}\right|-25 \leq 26$, we have by (2.35) and (2.36)

$$
I_{3} \leq C\|v\|_{W^{25,220 / 3}}\|w\|_{W^{26,6 /(1-2 \varepsilon)}}^{1-a}\|w\|_{H^{52}}^{a}
$$

Inequalities (2.33), (2.34) and (2.37) give us

$$
\begin{aligned}
& \sum_{|\alpha|=51}\left\|\partial_{x}^{\alpha}(v w)\right\|_{L^{2}} \\
& \leq C\left(\|v\|_{H^{51}}\|w\|_{W^{25,6 /(1-2 \varepsilon)}}\right. \\
& \left.\quad+\|v\|_{W^{25,220 / 3}}\|w\|_{W^{26,6 /(1-2 \varepsilon)}}^{1-a}\|w\|_{H^{52}}^{a}\right)
\end{aligned}
$$

On the other hand, we easily see by the Sobolev imbedding theorem that

$$
\begin{aligned}
\|v w\|_{L^{2}} & \leq\|v\|\left\|_{L^{3 /(1+\varepsilon)}}\right\| w \|_{L^{6 /(1-2 \varepsilon)}} \\
& \leq C\|v\|_{H^{1}}\|w\|_{L^{6 /(1-2 \varepsilon)}} .
\end{aligned}
$$

Since we have by interpolation

$$
\sum_{|\alpha|=k}\left\|\partial_{x}^{\alpha}(v w)\right\|_{L^{2}} \leq C\|v w\|_{L^{2}}^{1-k / 51}\left(\sum_{|\alpha|=51}\left\|\partial_{x}^{\alpha}(v w)\right\|_{L^{2}}\right)^{k / 51}
$$

for $1 \leq k \leq 50$, we obtain by (2.38) and (2.39)

$$
\begin{aligned}
\sum_{|\alpha| \leq 51}\left\|\partial_{x}^{\alpha}(v w)\right\|_{L^{2}} \leq & C\left(\|v\|_{H^{51}}\|w\|_{W^{25,6 /(1-2 \varepsilon)}}\right. \\
& \left.+\|v\|_{W^{25,220 / 3}}\|w\|_{W^{26,6 /(1-6 \varepsilon)}}^{1-a}\|w\|_{H^{52}}^{a}\right)
\end{aligned}
$$

which shows (2.30).

Vol. $12, n^{\circ} 4-1995$. 
Finally, the relation (2.31) is equivalent to

$$
\varepsilon \leq \frac{61}{5283}+\frac{220}{5283} \varepsilon^{2} .
$$

This relation holds if $0<\varepsilon<10^{-2}$. Thus, the proof of Lemma 2.5 is completed.

(Q.E.D.)

Concerning the unique local solvability of (1.1)-(1.3), we have the following lemma.

Lemma 2.6. - Let $N$ be the spatial dimensions.

(i) Assume $N=1$. Let $u_{0} \in H^{1}(\mathbf{R}), u_{1} \in L^{2}(\mathbf{R}), n_{0} \in L^{2}(\mathbf{R})$ and $n_{1} \in \dot{H}^{-1}(\mathbf{R})$. Then, there exists a $T>0$ depending only on $\left\|u_{0}\right\|_{H^{1}}$, $\left\|u_{1}\right\|_{L^{2}},\left\|n_{0}\right\|_{L^{2}}$ and $\left\|n_{1}\right\|_{\dot{H}^{-1}}$ such that (1.1)-(1.3) have the unique local solutions $(u(t), n(t))$ on $[0, T]$ satisfying

$$
\begin{aligned}
& u \in \bigcap_{j=0}^{2} C^{j}\left([0, T] ; H^{1-j}(\mathbf{R})\right), \\
& n \in \bigcap_{j=0}^{2} C^{j}\left([0, T] ; H^{-j}(\mathbf{R})\right), \\
& \partial_{t} n \in C\left([0, T] ; \dot{H}^{-1}(\mathbf{R})\right), \\
& \partial_{t}^{2} n \in C\left([0, T] ; \dot{H}^{-2}(\mathbf{R})\right) .
\end{aligned}
$$

In addition, if $u_{0} \in H^{m}(\mathbf{R}), u_{1} \in H^{m-1}(\mathbf{R}), n_{0} \in H^{m-1}(\mathbf{R})$ and $n_{1} \in H^{m-2}(\mathbf{R}) \cap \dot{H}^{-1}(\mathbf{R})$ for an integer $m$ with $m \geq 2$, then the above solutions $(u, n)$ satisfy

$$
\begin{aligned}
& u \in \bigcap_{j=0}^{m} C^{j}\left([0, T] ; H^{m-j}(\mathbf{R})\right), \\
& n \in \bigcap_{j=0}^{m} C^{j}\left([0, T] ; H^{m-1-j}(\mathbf{R})\right) .
\end{aligned}
$$

(ii) Assume $N=2$, 3. Let $u_{0} \in H^{2}\left(\mathbf{R}^{N}\right), u_{1} \in H^{1}\left(\mathbf{R}^{N}\right), n_{0} \in H^{1}\left(\mathbf{R}^{N}\right)$ and $n_{1} \in L^{2}\left(\mathbf{R}^{N}\right) \cap \dot{H}^{-1}\left(\mathbf{R}^{N}\right)$. Then, there exsits a $T>0$ depending only on $\left\|u_{0}\right\|_{H^{2}},\left\|u_{1}\right\|_{H^{1}},\left\|n_{0}\right\|_{H^{1}}$ and $\left\|n_{1}\right\|_{L^{2} \cap \dot{H}^{-1}}$ such that (1.1)-(1.3) have the unique local solutions $(u(t), n(t))$ on $[0, T]$ satisfying

$$
u \in \bigcap_{j=0}^{2} C^{j}\left([0, T] ; H^{2-j}\left(\mathbf{R}^{N}\right)\right),
$$




$$
\begin{aligned}
& n \in \bigcap_{j=0}^{2} C^{j}\left([0, T] ; H^{1-j}\left(\mathbf{R}^{N}\right)\right), \\
& \partial_{t} n \in C\left([0, T] ; \dot{H}^{-1}\left(\mathbf{R}^{N}\right)\right), \\
& \partial_{t}^{2} n \in C\left([0, T] ; \dot{H}^{-2}\left(\mathbf{R}^{N}\right)\right) .
\end{aligned}
$$

In addition, if $u_{0} \in H^{m}\left(\mathbf{R}^{N}\right), u_{1} \in H^{m-1}\left(\mathbf{R}^{N}\right), n_{0} \in H^{m-1}\left(\mathbf{R}^{N}\right)$ and $n_{1} \in H^{m-2}\left(\mathbf{R}^{N}\right) \cap \dot{H}^{-1}\left(\mathbf{R}^{N}\right)$ for an integer $m$ with $m \geq 3$, then the above solutions $(u, n)$ satisfy

$$
\begin{aligned}
& u \in \bigcap_{j=0}^{m} C^{j}\left([0, T] ; H^{m-j}\left(\mathbf{R}^{N}\right)\right), \\
& n \in \bigcap_{j=0}^{m} C^{j}\left([0, T] ; H^{m-1-j}\left(\mathbf{R}^{N}\right)\right) .
\end{aligned}
$$

(iii) Assume $N=3$. In addition to the assumptions in (ii), if $n_{0} \in \dot{H}^{-1}$ and $n_{1} \in \dot{H}^{-2}$ then the solution $n$ for the wave equation part of (1.1)-(1.3) given by (i) and (ii) satisfy

$$
\begin{aligned}
& n \in C^{1}\left([0, T] ; \dot{H}^{-1}\right), \\
& \partial_{t} n \in C^{1}\left([0, T] ; \dot{H}^{-2}\right) .
\end{aligned}
$$

Lemma 2.6 follows from the standard iteration argument. We note that $H^{1}(\mathbf{R}) \hookrightarrow L^{\infty}(\mathbf{R})$ for $N=1$ and $H^{2}\left(\mathbf{R}^{N}\right) \hookrightarrow L^{\infty}\left(\mathbf{R}^{N}\right)$ for $N=2,3$. We leave the proof of Lemma 2.6 to the reader.

Remark 2.2. - In Lemma 2.6, we need not assume that $\left(u_{0}, u_{1}\right)$ are real-valued. It follows from the uniqueness of solutions that if $n_{0}$ and $n_{1}$ are real-valued, $n(t, x)$ is a real-valued function.

If $n_{0}$ and $n_{1}$ are real-valued, then the solutions $(u, n)$ of $(1.1)-(1.3)$ formally satisfy the following energy identity:

$$
\begin{aligned}
& E\left(u(t), \partial_{t} u(t), n(t), \partial_{t} n(t)\right) \\
& \quad=E\left(u_{0}, u_{1}, n_{0}, n_{1}\right), t \geq 0
\end{aligned}
$$

where

$$
\begin{aligned}
E\left(u, \partial_{t} u, n, \partial_{t} n\right)= & \|\nabla u\|_{L^{2}}^{2}+\left\|\partial_{t} u\right\|_{L^{2}}^{2} \\
& +\|n\|_{L^{2}}^{2}+\left\|(-\triangle)^{-1 / 2} \partial_{t} n\right\|_{L^{2}}^{2}+\int_{\mathbf{R}^{N}} n|u|^{2} d x
\end{aligned}
$$

Vol. 12, n ${ }^{\circ}$ 4-1995. 
The global existence theorem for small initial data follows easily from Lemma 2.6 and (2.54), when $N=1,2$.

THEOREM 2.7. - Let $N$ be the spatial dimensions. Assume that $\left(u_{0}, u_{1}\right)$ and $\left(n_{0}, n_{1}\right)$ are complex-valued and real-valued functions on $\mathbf{R}^{N}$, respectively.

(i) Assume $N=1$. Let $u_{0} \in H^{1}(\mathbf{R}), u_{1} \in L^{2}(\mathbf{R}), n_{0} \in L^{2}(\mathbf{R})$ and $n_{1} \in \dot{H}^{-1}(\mathbf{R})$. Then, there exists a $\delta>0$ such that if

$$
\left\|u_{0}\right\|_{H^{1}}+\left\|u_{1}\right\|_{L^{2}}+\left\|n_{0}\right\|_{L^{2}}+\left\|n_{1}\right\|_{\dot{H}^{-1}} \leq \delta
$$

(1.1)-(1.3) have the unique global solutions $(u, n)$ satisfying (2.40)-(2.43) with the existence time interval $[0, T]$ replaced by $[0, \infty)$. In addition, if $u_{0} \in$ $H^{m}(\mathbf{R}), u_{1} \in H^{m-1}(\mathbf{R}), n_{0} \in H^{m-1}(\mathbf{R})$ and $n_{1} \in H^{m-2}(\mathbf{R}) \cap \dot{H}^{-1}(\mathbf{R})$ for an integer $m$ with $m \geq 2$, then the above global solutions $(u, n)$ satisfy (2.44) and (2.45) with the existence time interval $[0, T]$ replaced by $[0, \infty)$.

(ii) Assume $N=2$. Let $u_{0} \in H^{2}\left(\mathbf{R}^{2}\right), u_{1} \in H^{1}\left(\mathbf{R}^{2}\right), n_{0} \in H^{1}\left(\mathbf{R}^{2}\right)$ and $n_{1} \in L^{2}\left(\mathbf{R}^{2}\right) \cap \dot{H}^{-1}\left(\mathbf{R}^{2}\right)$. Then, there exists a $\delta>0$ such that if

$$
\left\|u_{0}\right\|_{H^{1}}+\left\|u_{1}\right\|_{L^{2}}+\left\|n_{0}\right\|_{L^{2}}+\left\|n_{1}\right\|_{\dot{H}^{-1}} \leq \delta
$$

(1.1)-(1.3) have the unique global solutions $(u, n)$ satisfying (2.46)(2.49) with the existence time interval $[0, T]$ replaced by $[0, \infty)$. In addition, if $u_{0} \in H^{m}\left(\mathbf{R}^{2}\right), u_{1} \in H^{m-1}\left(\mathbf{R}^{2}\right), n_{0} \in H^{m-1}\left(\mathbf{R}^{2}\right)$ and $n_{1} \in H^{m-2}\left(\mathbf{R}^{2}\right) \cap \dot{H}^{-1}\left(\mathbf{R}^{2}\right)$ for an integer $m$ with $m \geq 3$, then the above global solutions $(u, n)$ satisfy (2.50) and (2.51) with the existence time interval $[0, T]$ replaced by $[0, \infty)$.

Part (i) of Theorem 2.7 follows immediately from Lemma 2.6 (i) and (2.54). In the proof of Theorem 2.7 (ii), we need to use the logarithmic Sobolev inequality for $N=2$, together with Lemma 2.6 (ii) and (2.54) (see [3]).

Remark 2.3. - When $N=2$ or 3 , by (2.54) and the Galerkin method we obtain the weak global solutions $(u, n) \in L^{\infty}\left(0, \infty ; H^{1}\left(\mathbf{R}^{N}\right)\right) \oplus$ $L^{\infty}\left(0, \infty ; L^{2}\left(\mathbf{R}^{N}\right)\right)$ for any small initial data $\left(u_{0}, u_{1}, n_{0}, n_{1}\right) \in H^{1}\left(\mathbf{R}^{N}\right) \oplus$ $L^{2}\left(\mathbf{R}^{N}\right) \oplus L^{2}\left(\mathbf{R}^{N}\right) \oplus \dot{H}^{-1}\left(\mathbf{R}^{N}\right)$. However, the uniqueness of the above solutions in the energy class is not yet known.

\section{PROOFS OF MAIN RESULTS}

In this section we describe the proofs of Theorem 1.1 and Corollary 1.2. 
We first find the transformation which transforms (1.1)-(1.2) into a new system with cubic nonlinearity. Following Shatah [12], we introduce the new unknown functions $v(t, x)$ and $m(t, x)$ :

$$
\begin{aligned}
& v=u-\left[n, G_{1}, u\right]-\left[\partial_{t} n, G_{2}, \partial_{t} u\right], \\
& m=n-\left[u, H_{1}, u\right]-\left[\partial_{t} u, H_{2}, \partial_{t} u\right],
\end{aligned}
$$

where $G_{i}$ and $H_{i}, i=1,2$ are the kernel distributions to be determined later. Here and hereafter the time variable $t$ is omitted when this causes no confusion. A simple calculation gives us

$$
\begin{aligned}
\partial_{t}^{2} v= & \partial_{t}^{2} u-\left[\Delta n, G_{1}, u\right]-2\left[\partial_{t} n, G_{1}, \partial_{t} u\right] \\
& -\left[n, G_{1}, \triangle u-u\right]-\left[\triangle \partial_{t} n, G_{2}, \partial_{t} u\right]-2\left[\triangle n, G_{2}, \triangle u-u\right] \\
& -\left[\partial_{t} n, G_{2}, \triangle \partial_{t} u-\partial_{t} u\right] \\
& +F_{1}\left(u, \partial_{t} u, n, \partial_{t} n\right)+F_{2}\left(u, \partial_{t} u, n, \partial_{t} n\right)
\end{aligned}
$$

where

$$
\begin{aligned}
F_{1}\left(u, \partial_{t} u, n, \partial_{t} n\right)= & -\left[\Delta|u|^{2}, G_{1}, u\right] \\
& -\left[n, G_{1}, n u\right]-2\left[\triangle n, G_{2}, n u\right] \\
& -2\left[\Delta \operatorname{Re}\left(\bar{u} \partial_{t} u\right), G_{2}, \partial_{t} u\right] \\
& -2\left[\triangle|u|^{2}, G_{2}, \triangle u\right]+2\left[\Delta|u|^{2}, G_{2}, u\right] \\
& -\left[\partial_{t} n, G_{2}, u \partial_{t} n\right]-\left[\partial_{t} n, G_{2}, n \partial_{t} u\right] \\
F_{2}\left(u, \partial_{t} u, n, \partial_{t} n\right)= & -2\left[\Delta|u|^{2}, G_{2}, n u\right]
\end{aligned}
$$

Moreover, we have

$$
\begin{aligned}
(-\Delta+1) v= & -\Delta u+u+\left[\triangle n, G_{1}, u\right] \\
& +2 \sum_{j=1}^{3}\left[\partial_{j} n, G_{1}, \partial_{j} u\right]+\left[n, G_{1}, \Delta u\right] \\
& -\left[n, G_{1}, u\right]+\left[\triangle \partial_{t} n, G_{2}, \partial_{t} u\right] \\
& +2 \sum_{j=1}^{3}\left[\partial_{j} \partial_{t} n, G_{2}, \partial_{j} \partial_{t} u\right]+\left[\partial_{t} n, G_{2}, \triangle \partial_{t} u\right] \\
& -\left[\partial_{t} n, G_{2}, \partial_{t} u\right] .
\end{aligned}
$$


Therefore, by (3.3), (3.6) and (1.2) we obtain

$$
\begin{aligned}
\partial_{t}^{2} v-\triangle v+v= & -n u-2\left[\Delta n, G_{2}, \Delta u\right] \\
& +2\left[\triangle n, G_{2}, u\right]+2 \sum_{j=1}^{3}\left[\partial_{j} n, G_{1}, \partial_{j} u\right] \\
& -2\left[\partial_{t} n, G_{1}, \partial_{t} u\right]+2 \sum_{j=1}^{3}\left[\partial_{j} \partial_{t} n, G_{2}, \partial_{j} \partial_{t} u\right] \\
& +F_{1}\left(u, \partial_{t} u, n, \partial_{t} n\right)+F_{2}\left(u, \partial_{t} u, n, \partial_{t} n\right)
\end{aligned}
$$

Since $F_{1}$ is cubic and $F_{2}$ is quartic, $F_{1}$ and $F_{2}$ cause no trouble as long as we consider solving (1.1)-(1.3) around zero. It is the quadratic terms that cause trouble. Accordingly, we choose the kernel distributions $G_{1}$ and $G_{2}$ so that all quadratic terms in (3.7) cancel out:

$$
\begin{aligned}
& -n u-2\left[\Delta n, G_{2}, \Delta u\right]+2\left[\triangle n, G_{2}, u\right] \\
& \quad+2 \sum_{j=1}^{3}\left[\partial_{j} n, G_{1}, \partial_{j} u\right]-2\left[\partial_{t} n, G_{1}, \partial_{t} u\right] \\
& \quad+2 \sum_{j=1}^{3}\left[\partial_{j} \partial_{t} n, G_{2}, \partial_{j} \partial_{t} u\right]=0 .
\end{aligned}
$$

We can write

$$
(n u)(x)=\iint_{\mathbf{R}^{3} \times \mathbf{R}^{3}} n(y) \delta(x-y) \delta(x-z) u(z) d y d z,
$$

where $\delta$ is the Dirac delta function on $\mathbf{R}^{3}$. Therefore, we take the Fourier transform of (3.8) to obtain

$$
\begin{aligned}
& \left\{-1-2|p|^{2}|q|^{2} \hat{G}_{2}(p, q)-2|p|^{2} \hat{G}_{2}(p, q)\right. \\
& \left.\quad-2 p \cdot q \hat{G}_{1}(p, q)\right\} \hat{n}(p) \hat{u}(q) \\
& \quad+\left\{-\hat{G}_{1}(p, q)-p \cdot q \hat{G}_{2}(p, q)\right\} \partial_{t} \hat{n}(p) \partial_{t} \hat{u}(q)=0 .
\end{aligned}
$$

In order that (3.9) holds for all $\hat{u}, \partial_{t} \hat{u}, \hat{n}$ and $\partial_{t} \hat{n}$, the kernel distributions $\hat{G}_{1}$ and $\hat{G}_{2}$ must satisfy

$$
\begin{aligned}
& -1-2|p|^{2}|q|^{2} \hat{G}_{2}(p, q)-2|p|^{2} \hat{G}_{2}(p, q)-2 p \cdot q \hat{G}_{1}(p, q)=0, \\
& -\hat{G}_{1}(p, q)+p \cdot q \hat{G}_{2}(p, q)=0,
\end{aligned}
$$


which imply

$$
\begin{aligned}
& \hat{G}_{1}(p, q)=\frac{p \cdot q}{2\left\{|p|^{2}|q|^{2}-(p \cdot q)^{2}+|p|^{2}\right\}} \\
& \hat{G}_{2}(p, q)=\frac{-1}{2\left\{|p|^{2}|q|^{2}-(p \cdot q)^{2}+|p|^{2}\right\}}
\end{aligned}
$$

The same procedure as above yields

$$
\begin{aligned}
& \hat{H}_{1}(p, q)=\frac{(p \cdot q-1)|p+q|^{2}}{2\left\{|p|^{2}|q|^{2}-(p \cdot q)^{2}+|p+q|^{2}\right\}}, \\
& \hat{H}_{2}(p, q)=\frac{-|p+q|^{2}}{2\left\{|p|^{2}|q|^{2}-(p \cdot q)^{2}+|p+q|^{2}\right\}} .
\end{aligned}
$$

Formally, the functions $v$ and $m$ given by the transformation (3.1)-(3.2) with (3.10)-(3.13) satisfy the following new system with the nonlinearity higher than quadratic:

$$
\begin{aligned}
& \partial_{t}^{2} v-\Delta v+v=F_{1}\left(u, \partial_{t} u, n, \partial_{t} n\right) \\
& \quad+F_{2}\left(u, \partial_{t} u, n, \partial_{t} n\right), t>0, x \in \mathbf{R}^{3} \\
& \partial_{t}^{2} m-\Delta m=F_{3}\left(u, \partial_{t} u, n, \partial_{t} n\right) \\
& \quad+F_{4}\left(u, \partial_{t} u, n, \partial_{t} n\right), t>0, x \in \mathbf{R}^{3}
\end{aligned}
$$

where $F_{1}$ and $F_{2}$ are defined in (3.4) and (3.5), respectively, and

$$
\begin{aligned}
& F_{3}\left(u, \partial_{t} u, n, \partial_{t} n\right)=-\left[n u, H_{1}, u\right] \\
& \quad-\left[u, H_{1}, n u\right]-\left[u \partial_{t} n, H_{2}, \partial_{t} u\right] \\
& \quad-\left[n \partial_{t} u, H_{2}, \partial_{t} u\right]-2\left[n u, H_{2}, \triangle u\right] \\
& \quad+2\left[n u, H_{2}, u\right]-2\left[\triangle u, H_{2}, n u\right] \\
& \quad+2\left[u, H_{2}, n u\right]-\left[\partial_{t} u, H_{2}, u \partial_{t} n\right] \\
& \quad-\left[\partial_{t} u, H_{2}, n \partial_{t} u\right], \\
& F_{4}\left(u, \partial_{t} u, n, \partial_{t} n\right)=-2\left[n u, H_{2}, n u\right] .
\end{aligned}
$$

However, $\hat{G}_{1}(p, q)$ and $\hat{G}_{2}(p, q)$ have the singularity near $p=0$. In addition, $\hat{H}_{1}(p, q)$ and $\hat{H}_{2}(p, q)$ are not regular near $p=-q$, although they are locally bounded near $p=-q$. These singularities make our problem difficult. In Vol. $12, n^{\circ} 4-1995$. 
order to overcome this difficulty, we use Lemma 2.2, Corollary 2.3 and the special property of the system (1.1)-(1.2).

We show the following lemma, which will be useful in deriving the decay estimates of $v$ and $m$.

LEMMA 3.1. - (i) Let $k$ be an arbitrary nonnegative integer and let $0<\varepsilon<1 / 2$. We put $\tilde{k}_{j}=\max (k+9-j, 10-j)$ for $j=1,2$. Then,

$$
\begin{aligned}
& \sum_{|\alpha|=k}\left\|\partial_{x}^{\alpha}\left[\omega_{0}^{j}(u v), G_{j}, w\right]\right\|_{L^{6 /(5+2 \varepsilon)}} \\
& \leq C\left\{\|u\|_{W[(k+2) / 2], 6 /(1-2 \varepsilon)}\|v\|_{H^{k+2}}\right. \\
& \left.\quad+\|u\|_{H^{k+2}}\|v\|_{\left.W^{[(k+2) / 2], 6 /(1-2 \varepsilon)}\right\}}\right\} \mid w \|_{H^{k_{j}}}
\end{aligned}
$$

for $j=1,2$, where $C$ depends only on $k$ and $\varepsilon$.

(ii) Let $k$ be an arbitrary nonnegative integer, and let $0<\varepsilon<10^{-2}$ and $0<\eta<1 / 2$. Then

$$
\begin{aligned}
\sum_{|\alpha| \leq k} & \left\{\left\|\omega_{0}^{\eta} \partial_{x}^{\alpha}\left[u v, H_{j}, w\right]\right\|_{L^{220 / 217}}\right. \\
& \left.+\left\|\omega_{0}^{\eta} \partial_{x}^{\alpha}\left[w, H_{j}, u v\right]\right\|_{L^{220 / 217}}\right\} \\
\leq & C\left\{\|u\|_{H^{k+12-j}}\|v\|_{H^{k+12-j}}\|w\|_{W[(k+10) / 2]+2-j, 6 /(1-2 \epsilon)}\right. \\
& \left.+\|u\|_{H^{[(k+10) / 2]+3-j} \|}\|v\|_{W^{[(k+10) / 2]+2-j, 6 /(1-2 \varepsilon)} \|}\|w\|_{H^{k+12-j}}\right\}
\end{aligned}
$$

for $j=1,2$, where $C$ depends only on $k, \varepsilon$ and $\eta$.

(iii) Let $k$ be an arbitrary nonnegative integer and let $0<\varepsilon<1 / 2$. Then,

$$
\begin{aligned}
& \left\|\omega_{0}^{(1+4 \varepsilon) / 3} \partial_{t}^{j} \int_{0}^{t}\left(\omega_{0}^{-1} \sin \omega_{0}(t-s)\right)|u(s)|^{2} d s\right\|_{W^{k, 3 /(1+\varepsilon)}} \\
& \leq C(1+t)^{-(1-2 \varepsilon) / 3}\left\{\sup _{0 \leq s \leq t}(1+s)^{1+\varepsilon}\|u(s)\|_{\left.W^{[(k+j) / 2], 6 /(1-2 \varepsilon)}\right\}}\right\} \\
& \quad \times\left\{\sup _{0 \leq s \leq t}\|u(s)\|_{H^{k+j}}\right\}, \quad t \geq 0,
\end{aligned}
$$

for $j=0,1$, where $C$ depends only on $\varepsilon$ and $k$.

Proof. - (i) We first show (i). Suppose $k \geq 1$. Let $K_{j}(x, y), j=1,2$ be the kernel distributions on $\mathbf{R}^{3} \times \mathbf{R}^{3}$ such that

$$
\hat{K}_{j}(p, q)=\langle p\rangle^{-2}|p|^{1 / 2-\varepsilon} \hat{G}_{j}(p, q)\langle q\rangle^{-9+j}
$$

for $j=1,2$, and let $\alpha$ be any multi-index with $|\alpha|=k$. We have by Lemmas 2.2 and 2.4 (i) 
(3.18) $\left\|\partial_{x}^{\alpha}\left[\omega_{0}^{j}(u v), G_{j}, w\right]\right\|_{L^{6 /(5+2 \varepsilon)}}$

$$
\begin{aligned}
\leq & C \sum_{\left|\alpha_{1}\right|+\left|\alpha_{2}\right|=k}\left\|\left[\partial_{x}^{\alpha_{1}} \omega_{0}^{-1 / 2+\varepsilon} \omega^{2}(u v), K_{j}, \partial_{x}^{\alpha_{2}} \omega^{9-j} w\right]\right\|_{L^{6 /(5+2 \varepsilon)}} \\
\leq & C \sum_{\substack{\left|\alpha_{1}\right|+\left|\alpha_{2}\right|=k \\
\left|\alpha_{2}\right| \geq 1}}\left\|\omega_{0}^{-1 / 2+\varepsilon} \partial_{x}^{\alpha_{1}} \omega^{2}(u v)\right\|_{L^{3 /(1+\varepsilon)}}\left\|\partial_{x}^{\alpha_{2}} \omega^{9-j} w\right\|_{L^{2}} \\
& +\left.C \sum_{\substack{\left|\alpha_{1}\right|=k \\
\left|\alpha_{2}\right|=0}}\left\|\omega_{0}^{-1 / 2+\varepsilon} \partial_{x}^{\alpha_{1}} \omega^{2}(u v)\right\|\right|_{L^{2}}\left\|\omega^{9-j} w\right\|_{L^{3 /(1+\varepsilon)}} \\
= & I_{1}+I_{2}
\end{aligned}
$$

for $j=1,2$.

Since $\dot{H}^{1 / 2-\varepsilon} \hookrightarrow L^{3 /(1+\varepsilon)}$ and $H^{1} \hookrightarrow L^{3 /(1+\varepsilon)}$ for $0<\varepsilon<1 / 2$, we have

$$
\begin{aligned}
I_{1} \leq & C \sum_{\substack{\left|\alpha_{1}\right|+\left|\alpha_{2}\right|=k \\
\left|\alpha_{2}\right| \geq 1}}\left\|\partial_{x}^{\alpha_{1}} \omega^{2}(u v)\right\|_{L^{2}}\left\|\partial_{x}^{\alpha_{2}} \omega^{9-j} w\right\|_{L^{2}} \\
\leq & C \sum_{\substack{\left|\beta_{1}\right|+\left|\beta_{2}\right| \leq(k-1)+2 \\
\leq}}\left\|\sum_{\substack{\left|\beta_{1}\right|+\left|\beta_{2}\right| \leq k+1 \\
\left|\beta_{1}\right| \leq\left|\beta_{2}\right|}}\right\| \partial_{x}^{\beta_{1}} u \partial_{x}^{\beta_{2}} v\left\|L_{L^{2}}^{\beta_{1}} u\right\|_{L^{6 /(1-2 \varepsilon)}}\|\|_{H^{k+9-j}}\left\|\partial_{x}^{\beta_{2}} v\right\|_{L^{3 /(1+\varepsilon)}} \\
& \left.+\sum_{\substack{\left|\beta_{1}\right|+\left|\beta_{2}\right| \leq k+1 \\
\left|\beta_{1}\right|>\left|\beta_{2}\right|}}\left\|\partial_{x}^{\beta_{1}} u\right\|_{L^{3 /(1+\varepsilon)}}\left\|\partial_{x}^{\beta_{2}} v\right\|_{L^{6 /(1-2 \varepsilon)}}\right\}\|w\|_{H^{k+9-j}} \\
& C\left\{\|u\|_{W^{[(k+1) / 2], 6 /(1-6 \varepsilon)} \|}\|v\|_{H^{k+2}}\right. \\
& \left.+\|u\| H_{H^{k+2}}\|v\| \|_{W((k+1) / 21,6 /(1-6 \varepsilon)}\right\}\|w\|_{H^{k+9-j} .} .
\end{aligned}
$$

On the other hand, since $\dot{H}^{1 / 2-\varepsilon, 3 /(2-\varepsilon)} \hookrightarrow L^{2}$ and $H^{1} \hookrightarrow L^{3 /(1+\varepsilon)}$ for $0<\varepsilon<1 / 2$, we have

$$
\begin{aligned}
(3.20) \quad I_{2} & \leq C \sum_{\left|\alpha_{1}\right|=k}\left\|\partial_{x}^{\alpha_{1}} \omega^{2}(u v)\right\|_{L^{3 /(2-\varepsilon)}}\left\|\omega^{9-j} w\right\|_{L^{3 /(1+\varepsilon)}} \\
& \leq C \sum_{\substack{\left|\beta_{1}\right|+\left|\beta_{2}\right| \leq k+2\\
}} \| C\left\{\partial_{x}^{\beta_{1}} u \partial_{x}^{\beta_{2}} v\left\|_{L^{3 /(2-\varepsilon)}}\right\| w \|_{H^{10-j}}\right. \\
& \sum \sum_{\substack{\left|\beta_{1}\right|+\left|\beta_{2}\right| \leq k+2 \\
\left|\beta_{1}\right| \leq\left|\beta_{2}\right|}}\left\|\partial_{x}^{\beta_{1}} u\right\|_{L^{6 /(1-2 \varepsilon)}}\left\|\partial_{x}^{\beta_{2}} v\right\|_{L^{2}}
\end{aligned}
$$

Vol. 12, $\mathrm{n}^{\circ} 4-1995$. 


$$
\begin{aligned}
& \left.\quad+\sum_{\substack{\left|\beta_{1}\right|+\left|\beta_{2}\right| \leq k+2 \\
\left|\beta_{1}\right|>\left|\beta_{2}\right|}}\left\|\partial_{x}^{\beta_{1}} u\right\| L_{L^{2}}\left\|\partial_{x}^{\beta_{2}} v\right\|_{L^{6 /(1-2 \varepsilon)}}\right\}\|w\|_{H^{10-j}} \\
& \leq C\left\{\|u\|_{W^{[(k+2) / 2\}, 6 /(1-2 \varepsilon)} \mid}\|v\|_{H^{k+2}}\right. \\
& \left.+\|u\|_{H^{k+2}}\|v\|_{W^{[(k+2) / 2], 6 /(1-2 \varepsilon)}}\right\}\|w\|_{H^{10-j}} .
\end{aligned}
$$

Accordingly, (3.18)-(3.20) show Lemma 3.1 (i) for $k \geq 1$. If $k=0, I_{1}$ vanishes and $I_{2}$ alone appears at the right hand side of (3.18). Therefore, Lemma 3.1 (i) also holds for $k=0$.

(ii) We next prove (ii). Let $J_{j}(x, y), j=1,2$ be the kernel distributions on $\mathbf{R}^{3} \times \mathbf{R}^{3}$ such that

$$
\hat{J}_{j}(p, q)=\langle p+q\rangle^{-2}|p+q|^{\eta}\langle p\rangle^{j-2} \hat{H}_{j}(p, q)\langle q\rangle^{j-2}\langle p-q\rangle^{-8}
$$

for $j=1,2$. By Corollary 2.3 and Lemma 2.4 (i), (ii) we have

$$
\begin{aligned}
& \sum_{|\alpha| \leq k}\left\|\omega_{0}^{\eta} \partial_{x}^{\alpha}\left[u v, H_{j}, w\right]\right\|_{L^{220 / 217}} \\
& \leq C \sum_{\left|\alpha_{1}\right|+\left|\alpha_{2}\right| \leq k+10}\left\|\left[\omega^{2-j} \partial_{x}^{\alpha_{1}}(u v), J_{j}, \omega^{2-j} \partial_{x}^{\alpha_{2}} w\right]\right\|_{L^{220 / 217}} \\
& \leq C \sum_{\substack{\left|\alpha_{1}\right|+\left|\alpha_{2}\right| \leq k+10 \\
\left|\alpha_{2}\right| \leq[(k+10) / 2]}}\left\|\omega^{2-j} \partial_{x}^{\alpha_{1}}(u v)\right\|_{L^{660 /(541+214 \varepsilon)}} \\
& \times\left\|\omega^{2-j} \partial_{x}^{\alpha_{2}} w\right\|_{L^{6 /(1-2 \varepsilon)}} \\
& +C \sum_{\substack{\left|\alpha_{1}\right|+\left|\alpha_{2}\right| \leq k+10 \\
\left|\alpha_{2}\right|>[(k+10) / 2]}}\left\|\omega^{2-j} \partial_{x}^{\alpha_{1}}(u v)\right\|_{L^{220 / 107}}\left\|\omega^{2-j} \partial_{x}^{\alpha_{2}} w\right\|_{L^{2}} \\
& \leq C \sum_{|\alpha| \leq k+12-j}\left\|\partial_{x}^{\alpha}(u v)\right\|_{L^{660 /(541+214 \varepsilon)}} \\
& \times\|w\|_{W[(k+10) / 2]+2-j, 6 /(1-2 \varepsilon)} \\
& +C \sum_{\substack{\left|\alpha_{1}\right|+\left|\alpha_{2}\right| \leq k+10 \\
\left|\alpha_{2}\right| \leq\lfloor(k+10) / 2]}}\left\|\omega^{2-j} \partial_{x}^{\alpha_{1}}(u v)\right\|_{L^{220 / 107}}\left\|\omega^{2-j} \partial_{x}^{\alpha_{2}} w\right\|_{L^{2}} \\
& \leq C\left\{\sum_{\substack{\left|\alpha_{1}\right|+\left|\alpha_{2}\right| \leq k+12-j \\
\left|\alpha_{2}\right| \leq[(k+12-j) / 2]}}\left\|\partial_{x}^{\alpha_{1}} u \partial_{x}^{\alpha_{2}} v\right\|_{L^{660 /(541+214 \varepsilon)}}\right. \\
& \left.+\sum_{\substack{\left|\alpha_{1}\right|+\left|\alpha_{2}\right| \leq k+12-j \\
\left|\alpha_{2}\right|>[(k+12-j) / 2]}}\left\|\partial_{x}^{\alpha_{1}} u \partial_{x}^{\alpha_{2}} v\right\|_{\left.L^{660 /(541+214 \varepsilon)}\right\}}\right\}
\end{aligned}
$$




$$
\begin{gathered}
\times\|w\|_{W[(k+10) / 2]+2-j, 6 /(1-2 \varepsilon)} \\
+C\|u\|_{W[(k+10) / 2]+2-j, 660 /(211-220 \varepsilon)} \\
\times\|v\|_{W[(k+10) / 2]+2-j, 6 /(1-2 \varepsilon)}\|w\|_{H^{k+12-j}} \\
\leq C\left\{\|u\|_{H^{k+12-j}}\|v\|_{W[(k+12-j) / 2], 660 /(211-220 \varepsilon)}\right. \\
+\|u\|_{W}\{(k+12-j) / 2], 660 /(211-220 \varepsilon) \\
\\
\left.\times\|v\|_{H^{k+12-j}}\right\} \\
+C\|u\|_{W[(k+10) / 2]+2-j, 6 /(1-2 \varepsilon)} \\
\times\|v\|_{W[(k+10) / 2]+2]+2-j, 660 /(211-220 \varepsilon)}
\end{gathered}
$$

At the third inequality of (3.21) we have used the following relation:

$$
\begin{gathered}
\frac{1}{C}\left\|\omega^{k} u\right\|_{L^{p}} \leq\|u\|_{W^{k, p}} \leq C\left\|\omega^{k} u\right\|_{L^{p}}, \\
1<p<\infty, k \in \mathbf{N}
\end{gathered}
$$

(see, e.g., [2, Theorem 6.2.3 in Chapter 6]). Since $H^{1} \hookrightarrow L^{660 /(211-220 \varepsilon)}$ for $0<\varepsilon<10^{-2}$, by (3.21) we obtain Lemma 3.1 (ii) for $\left[u v, H_{j}, w\right]$. The proof for $\left[w, H_{j}, u v\right]$ is the same as above.

(iii) We show (iii). By Lemma 2.1 (ii) we have

$$
\begin{aligned}
& \left\|\partial_{t}^{j}\left(\omega_{0}^{-1} e^{i \omega_{0} t} \varphi\right)\right\|_{L^{3 /(1+\varepsilon)}} \\
& \quad \leq C|t|^{-(1-2 \varepsilon) / 3}\left\|\omega_{0}^{-(1+4 \varepsilon) / 3+j} \varphi\right\|_{L^{3 /(2-\varepsilon)}, t \neq 0, j=0,1}
\end{aligned}
$$

We prove (iii) only for $j=0$, because the proof for $j=1$ is the same.

We first note that

$$
\begin{aligned}
\left\|\omega_{0}^{s} f\right\|_{L^{p}} \leq C\|f\|_{W^{k, p}} \\
\\
\quad 0<s \leq k, k \in \mathbf{N}, 1<p<\infty .
\end{aligned}
$$

For (3.24), see, e.g., [2, Theorems 6.2.3 and 6.3.2 in Chapter 6]. By (3.23) Vol. $12, \mathbf{n}^{\circ} 4-1995$. 
and (3.24) we have

$$
\begin{aligned}
& \left\|\omega_{0}^{(1+4 \varepsilon) / 3} \int_{0}^{t}\left(\omega_{0}^{-1} \sin \omega_{0}(t-s)\right)|u(s)|^{2} d s\right\|_{W^{k, 3 /(1+\varepsilon)}} \\
& \leq C \int_{0}^{t}(t-s)^{-(1-2 \varepsilon) / 3}\left\|\left.u u(s)\right|^{2}\right\|_{W^{k, 3 /(2-\varepsilon)}} d s \\
& \leq C \int_{0}^{t}(t-s)^{-(1-2 \varepsilon) / 3}\|u(s)\|_{W^{[k / 2], 6 /(1-2 \varepsilon)} \|}\|u(s)\|_{H^{k}} d s \\
& \leq C \int_{0}^{t}(t-s)^{-(1-2 \varepsilon) / 3}(1+s)^{-1-\varepsilon} d s \\
& \quad \times\left\{\sup _{0 \leq s \leq t}(1+s)^{1+\varepsilon}\|u(s)\|_{\left.W^{[k / 2], 6 /(1-2 \varepsilon)}\right\}}\right. \\
& \quad \times\left\{\sup _{0 \leq s \leq t}\|u(s)\| H^{k}\right\} \\
& \leq C(1+t)^{-(1-2 \varepsilon) / 3}\left\{\sup _{0 \leq s \leq t}(1+s)^{1+\varepsilon}\|u(s)\|_{W^{[k / 2], 6 /(1-2 \varepsilon)}}\right\} \\
& \quad \times\left\{\sup _{0 \leq s \leq t}\|u(s)\|_{H^{k}}\right\}, t \geq 0 .
\end{aligned}
$$

This shows Lemma 3.1 (iii) for $j=0$. The proof for the case of $j=1$ is the same as above.

(Q.E.D.)

We are now in a position to prove Theorem 1.1.

Proof of Theorem 1.1. - For the proof of Theorem 1.1, we need derive the decay estimate and the energy estimate of solutions to (1.1)-(1.3). Let $(u, n)$ be the local solutions of (1.1)-(1.3) given by Lemma 2.6 (ii), (iii) and let $(v, m)$ be the functions given by the transformation (3.1)-(3.2). We put

$$
\begin{aligned}
X(t)= & \sup _{0 \leq s \leq t}\left\{\sum_{j=0}^{1}(1+s)^{1+\varepsilon}\left\|\partial_{s}^{j} u(s)\right\|_{W^{26-j, 6 /(1-2 \varepsilon)}}\right. \\
& +\sum_{j=0}^{1}\left\|\partial_{s}^{j} u(s)\right\|_{H^{52-j}}+\sum_{j=0}^{1}(1+s)^{107 / 110}\left\|\partial_{s}^{j} n(s)\right\|_{W^{25-j, 220 / 3}} \\
& \left.+\sum_{j=0}^{1}\left\|\partial_{s}^{j} n(s)\right\|_{H^{51-j}}+\sum_{j=0}^{1}\left\|\partial_{s}^{j} n(s)\right\|_{H^{-1-j}}\right\}
\end{aligned}
$$

for $t \geq 0$. Let $\delta$ be a positive constant satisfying the relation (1.6), which will be determined later.

We begin with the lemma concerning the decay estimate of the solutions. 
Lemma 3.2. - Let $0<\varepsilon<10^{-2}$. Then, the solutions $(u, n)$ of $(1.1)-(1.3)$ satisfy

$$
\begin{aligned}
& \sum_{j=0}^{1}(1+t)^{1+\varepsilon}\left\|\partial_{t}^{j} u(t)\right\|_{W^{26-j, 6 /(1-2 \varepsilon)}} \\
& \quad+\sum_{j=0}^{1}(1+t)^{107 / 110}\left\|\partial_{t}^{j} n(t)\right\|_{W^{25-j, 220 / 3}} \\
& \leq C\left\{\left(1+\delta+\delta^{2}\right) \delta+\delta X(t)+(1+\delta) X(t)^{2}+X(t)^{3}+X(t)^{4}\right\} \\
& t \geq 0,
\end{aligned}
$$

as long as the solutions $(u, n)$ exist. Here, $C$ does not depend on $\delta$ and $t$.

Proof. - We rewrite (3.14) and (3.15) as the following integral equations:

$$
\begin{aligned}
& v(t)=(\cos \omega t) v_{0}+\left(\omega^{-1} \sin \omega t\right) v_{1} \\
& \quad+\int_{0}^{t} \omega^{-1} \sin \omega(t-s)\left\{F_{1}\left(u, \partial_{s} u, n, \partial_{s} n\right)\right. \\
& \left.\quad+F_{2}\left(u, \partial_{s} u, n, \partial_{s} n\right)\right\} d s, t \geq 0 \\
& m(t)=\left(\cos \omega_{0} t\right) m_{0}+\left(\omega_{0}^{-1} \sin \omega_{0} t\right) m_{1} \\
& \quad+\int_{0}^{t} \omega_{0}^{-1} \sin \omega_{0}(t-s)\left\{F_{3}\left(u, \partial_{s} u, n, \partial_{s} n\right)\right. \\
& \left.\quad+F_{4}\left(u, \partial_{s} u, n, \partial_{s} n\right)\right\} d s, t \geq 0
\end{aligned}
$$

where $F_{1}, F_{2}, F_{3}$ and $F_{4}$ are defined in (3.4), (3.5), (3.16) and (3.17), respectively, and

$$
\begin{aligned}
& v_{0}=u_{0}-\left[n_{0}, G_{1}, u_{0}\right]-\left[n_{1}, G_{2}, u_{1}\right] \\
& v_{1}=u_{1}-\left[n_{1}, G_{1}, u_{0}\right]-\left[n_{0}, G_{1}, u_{1}\right] \\
& \quad-\left[\triangle\left(n_{0}+\left|u_{0}\right|^{2}\right), G_{2}, u_{1}\right]-\left[n_{1}, G_{2}, \triangle u_{0}-u_{0}+n_{0} u_{0}\right] \\
& m_{0}=n_{0}-\left[u_{0}, H_{1}, u_{0}\right]-\left[u_{1}, H_{2}, u_{1}\right] \\
& m_{1}=n_{1}-\left[u_{1}, H_{1}, u_{0}\right]-\left[u_{0}, H_{1}, u_{1}\right] \\
& \quad-\left[\triangle u_{0}-u_{0}+n_{0} u_{0}, H_{2}, u_{1}\right] \\
& \quad-\left[u_{1}, H_{2}, \triangle u_{0}-u_{0}+n_{0} u_{0}\right]
\end{aligned}
$$

We first show the decay estimate of $u$. For that purpose, we evaluate $v$ by using (3.25). By Lemma 2.1 (i), (3.22) and the assumption $0<\varepsilon<10^{-2}$, Vol. $12, n^{\circ} 4-1995$. 
we have

$$
\begin{aligned}
& \left\|\partial_{t}^{j}\left\{\left(\omega^{-1} \sin \omega t\right) \varphi\right\}\right\|_{L^{6 /(1-2 \varepsilon)}} \\
& \quad \leq C A(t)\|\varphi\|_{W^{1+j, 6 /(5+2 \varepsilon)}}, t>0
\end{aligned}
$$

for $j=0,1$, where

$$
A(t)=(1+t)^{-2(1+\varepsilon) / 3} t^{-(1+\varepsilon) / 3}
$$

and $C$ does not depend on $t$.

We take the $W^{26,6 /(1-2 \varepsilon)}$ norm of (3.25) to obtain by (3.32) and the Sobolev imbedding theorem

$$
\begin{aligned}
& \|v(t)\|_{W^{26,6 /(1-2 \varepsilon)} \leq} \leq C(1+t)^{-1-\varepsilon} \\
& \quad \times\left(\left\|v_{0}\right\|_{H^{28}}+\left\|v_{0}\right\|_{W^{28,6 /(5+2 \varepsilon)}}+\left\|v_{1}\right\|_{H^{27}}\right. \\
& \left.\quad+\left\|v_{1}\right\|_{\left.W^{27,6 /(5+2 \varepsilon)}\right)}\right)+C \int_{0}^{t} A(t-s) \\
& \quad \times\left\{\left\|F_{1}\left(u, \partial_{s} u, n, \partial_{s} n\right)\right\|_{W^{27,6 /(5+2 \varepsilon)}}\right. \\
& \left.\quad+\left\|F_{2}\left(u, \partial_{s} u, n, \partial_{s} n\right)\right\|_{W^{27,6 /(5+2 \varepsilon)}}\right\} d s .
\end{aligned}
$$

In the same way as (3.18) and (3.19), we have by Lemmas 2.2, 2.4 (i), the Sobolev imbedding theorem and (3.27)-(3.28)

$$
\begin{aligned}
& \text { (3.34) }\left\|v_{0}\right\|_{H^{28}}+\left\|v_{0}\right\|_{W^{28,6 /(5+2 \varepsilon)}}+\left\|v_{1}\right\|_{H^{27}} \\
& +\left\|v_{1}\right\|_{W^{27,6 /(5+2 e)}} \\
& \leq\left\|u_{0}\right\|_{H^{28}}+\left\|u_{0}\right\|_{W^{28,6 /(5+2 \varepsilon)}} \\
& +C\left\|v_{0}-u_{0}\right\|_{W^{30,6 /(5+2 \varepsilon)}} \\
& +\left\|u_{1}\right\|_{H^{27}}+\left\|u_{1}\right\|_{W^{27,6 /(5+2 \varepsilon)}} \\
& +C\left\|v_{1}-u_{1}\right\|_{W^{29,6 /(5+2 \varepsilon)}} \\
& \leq C \delta+C\left(\left\|n_{0}\right\|_{H^{31}}+\left\|n_{0}\right\|_{\dot{H}^{-1}}\right)\left\|u_{0}\right\|_{H^{38}} \\
& +C\left(\left\|n_{1}\right\|_{H^{30}}+\left\|n_{1}\right\|_{\dot{H}^{-2}}\right)\left\|u_{1}\right\|_{H^{37}} \\
& +C\left(\left\|n_{1}\right\|_{H^{31}}+\left\|n_{1}\right\|_{\dot{H}^{-1}}\right)\left\|u_{0}\right\|_{H^{38}} \\
& +C\left(\left\|n_{0}\right\|_{H^{31}}+\left\|n_{0}\right\|_{\dot{H}^{-1}}\right)\left\|u_{1}\right\|_{H^{38}} \\
& +C\left(\left\|n_{0}\right\|_{H^{31}}+\left\|u_{0}\right\|_{H^{31}}^{2}\right)\left\|u_{1}\right\|_{H^{37}} \\
& +C\left(\left\|n_{1}\right\|_{H^{30}}+\left\|n_{1}\right\|_{\dot{H}^{-2}}\right)\left(\left\|u_{0}\right\|_{H^{39}}+\left\|u_{0}\right\|_{H^{37}}\left\|n_{0}\right\|_{H^{37}}\right) \\
& \leq C\left(1+\delta+\delta^{2}\right) \delta \text {, }
\end{aligned}
$$


where $C$ does not depend on $\delta$.

We next evaluate the $W^{27,6 /(5+2 \varepsilon)}$ norm of $F_{1}$. By (3.4) we have

$$
\begin{aligned}
& \left\|F_{1}(s)\right\|_{W^{27,6 /(5+2 \varepsilon)}} \leq C\left\{\left\|\left[\triangle|u|^{2}, G_{1}, u\right]\right\|_{W^{27,6 /(5+2 \varepsilon)}}\right. \\
& +\left\|\left[n, G_{1}, n u\right]\right\|_{W^{27,6 /(5+2 \varepsilon)}}+\left\|\left[\triangle n, G_{2}, n u\right]\right\|_{W^{27,6 /(5+2 \varepsilon)}} \\
& +\left\|\left[\triangle \operatorname{Re}\left(\bar{u} \partial_{s} u\right), G_{2}, \partial_{s} u\right]\right\|_{W^{27,6 /(5+2 \varepsilon)}} \\
& +\left\|\left[\Delta|u|^{2}, G_{2}, \Delta u\right]\right\|_{W^{27,6 /(5+2 \varepsilon)}} \\
& +\left\|\left[\Delta|u|^{2}, G_{2}, u\right]\right\|_{W^{27,6 /(5+2 \varepsilon)}} \\
& +\left\|\left[\partial_{s} n, G_{2}, u \partial_{s} n\right]\right\|_{W^{27,6 /(5+2 \varepsilon)}} \\
& \left.+\left\|\left[\partial_{s} n, G_{2}, n \partial_{s} u\right]\right\|_{\left.W^{27,6 /(5+2 \varepsilon)}\right\}}\right\} \text {. }
\end{aligned}
$$

We evaluate only the second and fourth terms at the right hand side of (3.35), since the rest terms of (3.35) can be similarily estimated.

We first note that

$$
\begin{aligned}
n(s)= & \left(\cos \omega_{0} s\right) n_{0}+\left(\omega_{0}^{-1} \sin \omega_{0} s\right) n_{1} \\
& +\int_{0}^{s}\left(\omega_{0}^{-1} \sin \omega_{0}(s-\tau)\right) \triangle|u(\tau)|^{2} d \tau .
\end{aligned}
$$

By (3.36), (3.22)-(3.24), Lemmas 2.2, 2.4 (i), 3.1 (iii) and the Sobolev imbedding theorem, we have

$$
\begin{aligned}
& \left\|\left[n, G_{1}, n u\right](s)\right\|_{W^{27,6 /(5+2 \varepsilon)}} \\
& \leq\left.\left\|\left[\left(\cos \omega_{0} s\right) n_{0}, G_{1}, n u\right]\right\|\right|_{W^{27,6 /(5+2 \varepsilon)}} \\
& + \\
& +\left\|\left[\left(\omega_{0}^{-1} \sin \omega_{0} s\right) n_{1}, G_{1}, n u\right]\right\|_{W^{27,6 /(5+2 \varepsilon)}} \\
& +\|\left[\int_{0}^{s}\left(\omega_{0}^{-1} \sin \omega_{0}(s-\tau)\right) \omega_{0}^{2}\right. \\
& \left.\quad \times|u(\tau)|^{2} d \tau, G_{1}, n u\right] \|\left.\right|_{W^{27,6 /(5+2 \varepsilon)}} \\
& =\left\|\left[\omega_{0}^{-1-2 \varepsilon / 3} \omega^{2}\left(\cos \omega_{0} s\right) n_{0}, K_{1}, \omega^{8}(n u)\right]\right\|_{W^{27,6 /(5+2 \varepsilon)}} \\
& +\left\|\left[\omega_{0}^{-1-2 \varepsilon / 3} \omega^{2}\left(\omega_{0}^{-1} \sin \omega_{0} s\right) n_{1}, K_{1}, \omega^{8}(n u)\right]\right\|_{W^{27,6 /(5+2 \varepsilon)}} \\
& +\|\left[\omega_{0}^{1-2 \varepsilon / 3} \omega^{2} \int_{0}^{s}\left(\omega_{0}^{-1} \sin \omega_{0}(s-\tau)\right)\right. \\
& \left.\quad \quad \times|u(\tau)|^{2} d \tau, K_{1}, \omega^{8}(n u)\right] \|_{W^{27,6 /(5+2 \varepsilon)}} \\
& \leq C\left\{\left\|\omega_{0}^{-1-2 \varepsilon / 3} \omega^{2}\left(\cos \omega_{0} s\right) n_{0}\right\|_{W^{27,321 /(105-107 \varepsilon)}}\right.
\end{aligned}
$$

Vol. $12, \mathrm{n}^{\circ} 4-1995$. 


$$
\begin{aligned}
& +\left\|\omega_{0}^{-1-2 \varepsilon / 3} \omega^{2}\left(\omega_{0}^{-1} \sin \omega_{0} s\right) n_{1}\right\|_{W^{27,321 /(105-107 \varepsilon)}} \\
& +\| \omega_{0}^{1-2 \varepsilon / 3} \omega^{2} \int_{0}^{s}\left(\omega_{0}^{-1} \sin \omega_{0}(s-\tau)\right) \\
& \left.\times|u(\tau)|^{2} d \tau \|_{W^{27,321 /(105-107 \varepsilon)}}\right\} \\
& \times\left\|\omega^{8}(n u)\right\|_{W^{27,642 /(325+428 \varepsilon)}} \\
& \leq C\left\{\left\|\omega_{0}^{-105 / 107+4 \varepsilon / 3} \omega^{2}\left(\cos \omega_{0} s\right) n_{0}\right\|_{W^{27,3 /(1+\varepsilon)}}\right. \\
& +\left\|\omega_{0}^{-105 / 107+4 \varepsilon / 3} \omega^{2}\left(\omega_{0}^{-1} \sin \omega_{0} s\right) n_{1}\right\|_{W^{27,3 /(1+\varepsilon)}} \\
& +\| \omega_{0}^{220 / 321+(1+4 \varepsilon) / 3} \omega^{2} \int_{0}^{s}\left(\omega_{0}^{-1} \sin \omega_{0}(s-\tau)\right) \\
& \left.\times|u(\tau)|^{2} d \tau \|_{W^{27,3 /(1+\varepsilon)}}\right\} \\
& \times\left\|\omega^{8}(n u)\right\|_{W^{27,642 /(325+428 \varepsilon)}} \\
& \leq C(1+s)^{-(1-2 \varepsilon) / 3}\left\{\left\|\omega_{0}^{-101 / 321} \omega^{2} n_{0}\right\|_{W^{27,3 /(2-\varepsilon)}}\right. \\
& +\left\|\omega_{0}^{-105 / 107+4 \varepsilon / 3} \omega^{2} n_{0}\right\|_{H^{28}} \\
& +\left\|\omega_{0}^{-422 / 321} \omega^{2} n_{1}\right\|_{W^{27,3 /(2-\varepsilon)}} \\
& +\left\|\omega_{0}^{-212 / 107+4 \varepsilon / 3} \omega^{2} n_{1}\right\|_{H^{28}} \\
& +\left(\sup _{0 \leq \tau \leq s}(1+\tau)^{1+\varepsilon}\|u(\tau)\|_{\left.W^{15,6 /(1-2 \varepsilon)}\right)}\right. \\
& \left.\times\left(\sup _{0 \leq \tau \leq s}\|u(\tau)\|_{H^{30}}\right)\right\}\|n u\|_{W^{35,642 /(325+428 \varepsilon)},}
\end{aligned}
$$

where $K_{1}$ is defined as in Lemma 2.2 with $\eta=2 \varepsilon / 3$. At the last inequality but one we have used the fact that $\dot{H}^{2 / 107+2 \varepsilon, 3 /(1+\varepsilon)} \hookrightarrow L^{321 /(105-107 \varepsilon)}$ for $0<\varepsilon<10^{-2}$. The standard interpolation theorem yields

$$
\begin{aligned}
&\left\|\omega_{0}^{-101 / 321} \omega^{2} n_{0}\right\|_{W^{27,3 /(2-\varepsilon)}} \\
& \quad+\left\|\omega_{0}^{-105 / 107+4 \varepsilon / 3} \omega^{2} n_{0}\right\|_{H^{28}} \\
& \quad+\left\|\omega_{0}^{-422 / 321} \omega^{2} n_{1}\right\|_{W^{27,3 /(2-\varepsilon)}} \\
& \quad+\left\|\omega_{0}^{-212 / 107+4 \varepsilon / 3} \omega^{2} n_{1}\right\|_{H^{28}} \\
& \leq C\left\{\left\|n_{0}\right\|_{H^{30}}+\left\|n_{0}\right\|_{\dot{H}^{-1}}+\left\|n_{0}\right\|_{W^{29,220 / 217}}\right. \\
&\left.+\left\|n_{1}\right\| H_{H^{29}}+\left\|n_{1}\right\|_{\dot{H}^{-2}}+\left\|n_{1}\right\|_{W^{28,220 / 217}}\right\} \leq C \delta
\end{aligned}
$$

(see, e.g., [2, Theorem 6.4.5 (7) in Chapter 6]). A direct calculation and the Sobolev imbedding theorem give us 
(3.39) $\|n u\|_{W^{35,642 /(325+428 \varepsilon)}}$

$$
\begin{aligned}
\leq & C \sum_{\substack{\left|\alpha_{1}\right|+\left|\alpha_{2}\right| \leq 35 \\
\left|\alpha_{1}\right| \leq\left|\alpha_{2}\right|}}\left\|\partial_{x}^{\alpha_{1}} n \partial_{x}^{\alpha_{2}} u\right\|_{L^{642 /(325+428 \varepsilon)}} \\
& +C \sum_{\substack{\left|\alpha_{1}\right|+\left|\alpha_{2}\right| \leq 35 \\
\left|\alpha_{1}\right|>\left|\alpha_{2}\right|}}\left\|\partial_{x}^{\alpha_{1}} n \partial_{x}^{\alpha_{2}} u\right\|_{L^{642 /(325+428 c)}} \\
\leq & C\|n\|_{W^{17,220 / 3}}\|u\|_{W^{35,70620 /(34787+47080 \varepsilon)}} \\
& +C\|n\|_{\left.W^{35,70620 /(34787+47080 \varepsilon)}\right)}\|u\|_{W^{17,6 /(1-2 c)}} \\
\leq & C(1+s)^{-107 / 110}\left(\sup _{0 \leq \tau \leq s}(1+\tau)^{107 / 110}\|n(\tau)\|_{W^{17,220 / 3}}\right) \\
& \quad \times\left(\sup _{0 \leq \tau \leq s}\|u(\tau)\|_{H^{36}}\right) \\
& +C(1+s)^{-(1+\varepsilon)}\left(\sup _{0 \leq \tau \leq s}\|n(\tau)\|_{H^{36}}\right) \\
& \times\left(\sup _{0 \leq \tau \leq s}(1+\tau)^{1+\varepsilon}\|u(\tau)\|_{W^{17,6 /(1-2 \varepsilon)}}\right) .
\end{aligned}
$$

By (3.37)-(3.39) we obtain

$$
\begin{aligned}
& \left\|\left[n, G_{1}, n u\right](s)\right\|_{W^{27,6 /(5+2 c)}} \\
& \quad \leq C(1+s)^{-1-\varepsilon}\left\{\delta+X(s)^{2}\right\} X(s)^{2}, 0 \leq s \leq t .
\end{aligned}
$$

We next evaluate the fourth term at the right hand side of (3.35). By Lemma 3.1 (i) we have

$$
\begin{aligned}
\left\|\left[\Delta \operatorname{Re}\left(\bar{u} \partial_{s} u\right), G_{2}, \partial_{s} u\right](s)\right\|_{W^{27,6 /(5+2 \varepsilon)}} \\
\leq C\left\{\|u\|_{W^{14,6 /(1-2 \varepsilon)}}\left\|\partial_{s} u\right\|_{H^{29}}\right. \\
\quad+\|u\|_{H^{29}}\left\|\partial_{s} u\right\|_{\left.W^{14,6 /(1-2 \varepsilon)}\right\}\left\|\partial_{s} u\right\|_{H^{34}}} \\
\leq C(1+s)^{-1-\varepsilon} X(s)^{3}, 0 \leq s \leq t
\end{aligned}
$$

Since the other terms at the right hand side of (3.35) can be similarily evaluated, we obtain by (3.35), (3.40), (3.41) and (3.32)

$$
\begin{gathered}
\int_{0}^{t} A(t-s)\left\|F_{1}\left(u, \partial_{s} u, n, \partial_{s} n\right)\right\|_{W^{27,6 /(5+2 \epsilon)}} d s \\
\leq C(1+s)^{-1-\varepsilon}\left\{\delta+X(t)+X(t)^{2}\right\} X(t)^{2}
\end{gathered}
$$

where $C$ does not depend on $\delta$ and $t$.

Vol. $12, n^{\circ} 4-1995$. 
In the same way as the case of $F_{1}$, we obtain

$$
\begin{aligned}
& \int_{0}^{t} A(t-s)\left\|F_{2}\left(u, \partial_{s} u, n, \partial_{s} n\right)\right\|_{W^{27,6 /(5+2 \varepsilon)} d s} \\
& \quad \leq C(1+s) X(t)^{4}
\end{aligned}
$$

where $C$ does not depend on $\delta$ and $t$.

Therefore, (3.33), (3.34), (3.42) and (3.43) yield

$$
\begin{aligned}
& \|v(t)\|_{W^{26,6 /(1-6 \varepsilon)} \leq C(1+t)^{-1-\varepsilon}}\left\{\left(1+\delta+\delta^{2}\right) \delta\right. \\
& \left.+\delta X(t)^{2}+X(t)^{3}+X(t)^{4}\right\}, t \geq 0,
\end{aligned}
$$

where $C$ does not depend on $\delta$ and $t$. Since the $W^{25,6 /(1-2 \varepsilon)}$ norm of $\partial_{t} v$ can be similarily evaluated, we obtain

$$
\begin{aligned}
& \sum_{j=0}^{1}(1+t)^{1+\varepsilon}\left\|\partial_{t}^{j} v(t)\right\|_{W^{26-j, 6 /(1-2 \varepsilon)}} \\
& \quad \leq C\left\{\left(1+\delta+\delta^{2}\right) \delta+\delta X(t)^{2}+X(t)^{3}+X(t)^{4}\right\}
\end{aligned}
$$

as long as the solutions $(u, n)$ of (1.1)-(1.3) exist, where $C$ does not depend on $\delta$ and $t$.

It remains to evaluate the second and third terms at the right hand side of (3.1) in order to obtain the decay estimate of $u$.

By (3.36) and Lemmas 2.1, 2.4 (i) we have

$$
\begin{aligned}
&\left\|\left[n, G_{1}, u\right]\right\|_{W^{26,6 /(1-2 \varepsilon)}} \\
& \leq\left\|\left[\omega_{0}^{-1-\varepsilon / 2} \omega^{2}\left(\cos \omega_{0} t\right) n_{0}, K_{1}, \omega^{8} u\right]\right\|_{W^{26,6 /(1-2 \varepsilon)}} \\
&+\left\|\left[\omega_{0}^{-1-\varepsilon / 2} \omega^{2}\left(\omega_{0}^{-1} \sin \omega_{0} t\right) n_{1}, K_{1}, \omega^{8} u\right]\right\|_{W^{26,6 /(1-2 \varepsilon)}} \\
&+\|\left[\omega_{0}^{-1-\varepsilon / 2} \omega^{2} \int_{0}^{t}\left(\omega_{0}^{-1} \sin \omega_{0}(t-s)\right)\right. \\
&\left.\times \triangle|u(s)|^{2} d s, K_{1}, \omega^{8} u\right] \|_{W^{26,6 /(1-2 \varepsilon)}} \\
& \leq C\left\{\left\|\omega_{0}^{-1-\varepsilon / 2} \omega^{2}\left(\cos \omega_{0} t\right) n_{0}\right\|_{W^{26,6 / \varepsilon}}\right. \\
&+\left\|\omega_{0}^{-1-\varepsilon / 2} \omega^{2}\left(\omega_{0}^{-1} \sin \omega_{0} t\right) n_{1}\right\|_{W^{26,6 / \varepsilon}} \\
&\left.+\left\|\omega_{0}^{-1-\varepsilon / 2} \omega^{2} \int_{0}^{t}\left(\omega_{0}^{-1} \sin \omega_{0}(t-s)\right) \triangle|u(s)|^{2} d s\right\|_{W^{26,6 / \varepsilon}}\right\}
\end{aligned}
$$




$$
\begin{aligned}
& \times\left\|\omega^{8} u\right\|_{W^{26,6 /(1-3 \varepsilon)}} \\
& \leq C\left\{\left\|\omega^{2}\left(\cos \omega_{0} t\right) n_{0}\right\|_{W^{26,3 /(1+\varepsilon)}}\right. \\
& +\left\|\omega^{2}\left(\omega_{0}^{-1} \sin \omega_{0} t\right) n_{1}\right\|_{W^{26,3 /(1+\varepsilon)}} \\
& \left.+\left\|\omega^{2} \int_{0}^{t}\left(\omega_{0}^{-1} \sin \omega_{0}(t-s)\right) \Delta|u(s)|^{2} d s\right\|_{W^{26,3 /(1+\varepsilon)}}\right\} \\
& \quad \times\left\|\omega^{8} u\right\|_{W^{26,6 /(1-3 \varepsilon)}},
\end{aligned}
$$

where $K_{1}$ is defined as in Lemma 2.1 with $\eta=\varepsilon / 2$. At the last inequality of (3.45) we have used the fact that $\dot{H}^{1+\varepsilon / 2,3 /(1+\varepsilon)} \hookrightarrow L^{6 / \varepsilon}$. By (3.23), the Sobolev imbedding theorem and the interpolation, we have

$$
\begin{aligned}
&\left\|\omega^{2} \partial_{t}^{1-j}\left\{\left(\omega_{0}^{-1} \sin \omega_{0} t\right) n_{j}\right\}\right\|_{W^{26,3 /(1+\epsilon)}} \\
& \leq C(1+t)^{-(1-2 \varepsilon) / 3}\left(\left\|n_{j}\right\|_{H^{28-j}}+\left\|n_{j}\right\|_{\dot{H}^{-j}}\right. \\
&\left.+\left\|\omega_{0}^{-(1+4 \varepsilon) / 3+1-j} n_{j}\right\|_{W^{28,3 /(2-\varepsilon)}}\right) \\
& \leq C(1+t)^{-(1-2 \varepsilon) / 3}\left(\left\|n_{j}\right\|_{H^{29}}+\left\|n_{j}\right\|_{\dot{H}^{-1-j}}\right. \\
&\left.+\left\|n_{j}\right\|_{W^{29-j, 220 / 217}}\right) \\
& \leq C(1+t)^{-(1-2 \varepsilon) / 3} \delta, j=0,1
\end{aligned}
$$

where $C$ does not depend on $\delta$ and $t$. Furthermore, Lemma 3.1 (iii), (3.22) and (3.24) yield

$$
\begin{aligned}
& \left\|\omega^{2} \int_{0}^{t}\left(\omega_{0}^{-1} \sin \omega_{0}(t-s)\right) \Delta|u(s)|^{2} d s\right\|_{W^{26,3 /(1+\varepsilon)}} \\
& \leq C\left\|\omega_{0}^{(1+4 \varepsilon) / 3} \int_{0}^{t}\left(\omega_{0}^{-1} \sin \omega_{0}(t-s)\right)|u(s)|^{2} d s\right\|_{W^{30,3 /(1+\varepsilon)}} \\
& \leq C(1+t)^{-(1-2 \varepsilon) / 3}\left\{\sup _{0 \leq s \leq t}\|u(s)\|_{\left.W^{15,6 /(1-2 \varepsilon)}\right\}}\right. \\
& \times\left\{\sup _{0 \leq s \leq t}\|u(s)\|_{H^{30,2}}\right\}
\end{aligned}
$$

On the other hand, (3.22) and the Gagliardo-Nirenberg inequality give us

$$
\begin{aligned}
& \left\|\omega^{8} u\right\|_{W^{26,6 /(1-3 \varepsilon)}} \leq C\|u\|_{W^{34,6 /(1-3 \epsilon)}} \\
& \leq C\|u\|_{W^{25,6 /(1-3 \varepsilon)}}+C \sum_{|\alpha|=8} \sum_{18 \leq|\beta| \leq 26}\left\|\partial_{x}^{\alpha}\left(\partial_{x}^{\beta} u\right)\right\|_{L^{6 /(1-3 \varepsilon)}} \\
& \leq C\|u\|_{W^{26,6 /(1-2 \varepsilon)}} \\
& \quad+C \sum_{18 \leq|\beta| \leq 26}\left\|\partial_{x}^{\beta} u\right\|_{H^{26}}^{b}\left\|\partial_{x}^{\beta} u\right\|_{L^{6 /(1-2 \varepsilon)}}^{1-b}
\end{aligned}
$$


where

$$
b=\frac{16+\varepsilon}{2(25-\varepsilon)} .
$$

For the proof of $(3.48)$, we need the following relation:

$$
\frac{8}{26} \leq b=\frac{16+\varepsilon}{2(25-\varepsilon)}
$$

which is satisfied for $\varepsilon>0$. If $0<\varepsilon<10^{-2}$, then we have

$$
(1-b)(1+\varepsilon) \geq \frac{2}{3}+\frac{5}{3} \varepsilon
$$

which is equivalent to $2+\varepsilon^{2} \geq 153 \varepsilon$. Therefore, by (3.48) we have

(3.49) $\left\|\omega^{8} u\right\|_{W^{26,6 /(1-3 \epsilon)}}$

$$
\begin{aligned}
& \leq C(1+t)^{-1-\varepsilon}\left\{\sup _{0 \leq s \leq t}(1+s)^{1+\varepsilon}\|u(s)\|_{\left.W^{26,6 /(1-2 \varepsilon)}\right\}}\right\} \\
& +C(1+t)^{-(2+5 \varepsilon) / 3}\left\{\sup _{0 \leq s \leq t}(1+s)^{1+\varepsilon}\|u(s)\|_{W^{26,6 /(1-2 \varepsilon)}}\right\}^{1-b} \\
& \quad \times\left\{\sup _{0 \leq s \leq t}\|u(s)\|_{H^{52}}\right\}^{b} .
\end{aligned}
$$

By (3.45)-(3.49) we obtain

$$
\begin{aligned}
& \left\|\left[n, G_{1}, u\right]\right\|_{W^{26,6 /(1-2 \varepsilon)}} \\
& \quad \leq C(1+t)^{-1-\varepsilon}\left\{\delta+X(t)^{2}\right\} X(t),
\end{aligned}
$$

where $C$ does not depend on $\delta$ and $t$. In the same way as above, we obtain

$$
\begin{aligned}
& \left\|\left[\partial_{t} n, G_{2}, \partial_{t} u\right]\right\|_{W^{26,6 /(1-2 \varepsilon)}} \\
& \quad \leq C(1+t)^{-1-\varepsilon}\left\{\delta+X(t)^{2}\right\} X(t),
\end{aligned}
$$

where $C$ does not depend on $\delta$ and $t$.

Accordingly, (3.1), (3.44), (3.50) and (3.51) yield

$$
\begin{aligned}
& (1+t)^{1+\varepsilon}\|u(t)\|_{W^{26,6 /(1-2 \varepsilon)}} \\
& \quad \leq C\left\{\left(1+\delta+\delta^{2}\right) \delta+\delta X(t)+\delta X(t)^{2}+X(t)^{3}+X(t)^{4}\right\}
\end{aligned}
$$


where $C$ does not depend on $\delta$ and $t$. Since the derivatives in $t$ of the second and third terms at the right hand side of (3.1) can be similarily evaluated, we finally obtain

$$
\begin{aligned}
& \sum_{j=0}^{1}(1+t)^{1+\epsilon}\left\|\partial_{t}^{j} u(t)\right\|_{W^{26-j, 6 /(1-2 \varepsilon)}} \\
& \quad \leq C\left\{\left(1+\delta+\delta^{2}\right) \delta+\delta X(t)+\delta X(t)^{2}+X(t)^{3}+X(t)^{4}\right\}, t \geq 0
\end{aligned}
$$

as long as the solutions $(u, n)$ of (1.1)-(1.3) exist, where $C$ does not depend on $\delta$ and $t$.

We next derive the decay estimate of $n$. For that purpose, we first evaluate $m$ by using (3.26). By Lemma 2.1 (ii), (3.22) and (3.24) we have

(3.53) $\left\|\partial_{t}^{j}\left\{\left(\omega_{0}^{-1} \sin \omega_{0} t\right) \varphi\right\}\right\|_{L^{220 / 3}}$

$$
\begin{aligned}
& \leq C|t|^{-107 / 110} \min \left(\|\varphi\|_{W^{1+j, 220 / 217}},\left\|\omega_{0}^{1 / 4} \varphi\right\|_{W^{1+j, 220 / 217}}\right), \\
& \quad t \neq 0, j=0,1 .
\end{aligned}
$$

We take the $W^{25,220 / 3}$ norm of (3.26) to obtain by (3.53) and the Sobolev imbedding theorem

$$
\begin{aligned}
& \|m(t)\|_{W^{25,220 / 3}} \leq C(1+t)^{-107 / 110} \\
& \quad \times\left\{\left\|n_{0}\right\|_{H^{27}}+\left\|n_{0}\right\|_{W^{27,220 / 217}}\right. \\
& \left.\quad+\left\|\omega_{0}^{-1} n_{1}\right\|_{H^{27}}+\left\|n_{1}\right\|_{W^{26,220 / 217}}\right\} \\
& \quad+C \int_{0}^{t}(t-s)^{-107 / 110}\left\{\left\|\omega_{0}^{1 / 4} F_{3}\left(u, \partial_{s} u, n, \partial_{s} n\right)\right\|_{W^{26,220 / 217}}\right. \\
& \left.\quad+\left\|\omega_{0}^{1 / 4} F_{4}\left(u, \partial_{s} u, n, \partial_{s} n\right)\right\|_{W^{26,220 / 217}}\right\} d s, t \geq 0 .
\end{aligned}
$$

By (3.16) and Lemma 3.1 (ii) with $\eta=\frac{1}{4}$, we have

$$
\begin{aligned}
\| \omega_{0}^{1 / 4} & F_{3}\left(u, \partial_{s} u, n, \partial_{s} n\right) \|_{W^{26,220 / 217}} \\
\leq & C\left\{\|n\|_{H^{37}}\|u\|_{H^{37}}\|u\|_{W^{19,6 /(1-2 \varepsilon)}}\right. \\
& \left.+\|n\|_{H^{20}}\|u\|_{W^{19,6 /(1-2 \varepsilon)}}\|u\|_{H^{37}}\right\} \\
& +C\left\{\left\|\partial_{s} n\right\|_{H^{36}}\|u\|_{H^{36}}\left\|\partial_{s} u\right\|_{W^{18,6 /(1-2 \varepsilon)}}\right. \\
& \left.+\left\|\partial_{s} n\right\|_{H^{19}}\|u\|_{W^{18,6 /(1-2 \varepsilon)}}\left\|\partial_{s} u\right\|_{H^{36}}\right\} \\
& +C\left\{\|n\|_{H^{36}}\left\|\partial_{s} u\right\|_{H^{36}}\left\|\partial_{s} u\right\|_{W^{18,6 /(1-2 \varepsilon)}}\right. \\
& \left.+\|n\| H_{H^{19}}\left\|\partial_{s} u\right\|_{W^{18,6 /(1-2 \varepsilon)}}\left\|\partial_{s} u\right\|_{H^{36}}\right\} \\
\leq & C(1+s)^{-1-\varepsilon} X(s)^{3}, 0 \leq s \leq t .
\end{aligned}
$$


Since $F_{4}$ is quartic, $F_{4}$ is easier to treat than $F_{3}$. Therefore, in the same way we obtain

$$
\begin{aligned}
& \left\|\omega_{0}^{1 / 4} F_{4}\left(u, \partial_{s} u, n, \partial_{s} n\right)\right\|_{W^{26,220 / 217}} \\
& \quad \leq C(1+s)^{-1-\varepsilon} X(s)^{4}, 0 \leq s \leq t .
\end{aligned}
$$

By the definition of $\delta$ we have

$$
\begin{aligned}
& \left\|n_{0}\right\|_{H^{27}}+\left\|n_{0}\right\|_{W^{27,220 / 217}} \\
& \quad+\left\|\omega_{0}^{-1} n_{1}\right\|_{H^{27}}+\left\|n_{1}\right\|_{W^{26,220 / 217}} \leq \delta .
\end{aligned}
$$

Accordingly, (3.54)-(3.57) yield

$$
\begin{aligned}
& \|m(t)\|_{W^{25,220 / 3}} \leq C(1+t)^{-107 / 110} \delta \\
& \quad+C \int_{0}^{t}(t-s)^{-107 / 110}(1+s)^{-1-\varepsilon} d s\left\{X(t)^{3}+X(t)^{4}\right\} \\
& \quad \leq C(1+t)^{-107 / 110}\left\{\delta+X(t)^{3}+X(t)^{4}\right\},
\end{aligned}
$$

where $C$ does not depend on $\delta$ and $t$. Since the $W^{24,220 / 3}$ norm of $\partial_{t} m$ can be similarily evaluated, we obtain

$$
\begin{aligned}
& \sum_{j=0}^{1}(1+t)^{107 / 110}\left\|\partial_{t} m(t)\right\|_{W^{25-j, 220 / 3}} \\
& \quad \leq C(1+t)^{-107 / 110}\left\{\delta+X(t)^{3}+X(t)^{4}\right\}, t \geq 0
\end{aligned}
$$

as long as the solutions $(u, n)$ of (1.1)-(1.3) exist, where $C$ does not depend on $\delta$ and $t$.

In order to derive the decay estimate of $n(t)$, it remains to evaluate the second and third terms at the right hand side of (3.2).

We put $\eta=(101-220 \varepsilon) / 220$. We first note that $\dot{H}^{3 / 2+\eta, 3 /(2-\varepsilon)} \hookrightarrow$ $L^{220 / 3}$ and $0<\eta<1 / 2$ for $0<\varepsilon<10^{-2}$. Therefore, by Corollary 2.3, Lemma 2.4 (i), (ii) and (3.24), we have

$$
\begin{aligned}
& \left\|\left[\partial_{t}^{j-1} u, H_{j}, \partial_{t}^{j-1} u\right]\right\|_{W^{25,220 / 3}} \\
& \quad \leq C\left\|\omega_{0}^{\eta}\left[\partial_{t}^{j-1} u, H_{j}, \partial_{t}^{j-1} u\right]\right\|_{W^{27,3 /(2-\varepsilon)}} \\
& \quad \leq C \sum_{\left|\alpha_{1}\right|+\left|\alpha_{2}\right| \leq 37}\left\|\left[\omega^{2-j} \partial_{x}^{\alpha_{1}} \partial_{t}^{j-1} u, J_{j}, \omega^{2-j} \partial_{x}^{\alpha_{2}} \partial_{t}^{j-1} u\right]\right\|_{L^{3 /(2-\varepsilon)}}
\end{aligned}
$$




$$
\begin{aligned}
\leq & C \sum_{\substack{\left|\alpha_{1}\right|+\left|\alpha_{2}\right| \leq 37 \\
\left|\alpha_{1}\right| \geq\left|\alpha_{2}\right|}}\left\|\omega^{2-j} \partial_{x}^{\alpha_{1}} \partial_{t}^{j-1} u\right\|_{L^{2}}\left\|\omega^{2-j} \partial_{x}^{\alpha_{2}} \partial_{t}^{j-1} u\right\|_{L^{6 /(1-2 \varepsilon)}} \\
& +C \sum_{\substack{\left|\alpha_{1}\right|+\left|\alpha_{2}\right| \leq 37 \\
\left|\alpha_{1}\right|<\left|\alpha_{2}\right|}}\left\|\omega^{2-j} \partial_{x}^{\alpha_{1}} \partial_{t}^{j-1} u\right\|_{L^{6 /(1-2 \varepsilon)}}\left\|\omega^{2-j} \partial_{x}^{\alpha_{2}} \partial_{t}^{j-1} u\right\|_{L^{2}} \\
& \leq C\left\|\omega^{2-j} \partial_{t}^{j-1} u\right\|_{H^{37}}\left\|\omega^{2-j} \partial_{t}^{j-1} u\right\|_{W^{18,6 /(1-2 \varepsilon)}} \\
& +C\left\|\omega^{2-j} \partial_{t}^{j-1} u\right\|_{W^{18,6 /(1-2 \varepsilon)}}\left\|\omega^{2-j} \partial_{t}^{j-1} u\right\|_{H^{37}} \\
\leq & C(1+t)^{-1-\varepsilon} X(t)^{2} \\
\leq & C(1+t)^{-107 / 110} X(t)^{2}, j=0,1
\end{aligned}
$$

where $J_{j}, j=0,1$ are defined as in Corollary 2.3 and $C$ does not depend on $\delta$ and $t$.

By (3.2), (3.58) and (3.59), we obtain

$$
\begin{aligned}
& \|n(t)\|_{W^{25,220 / 3} \leq C(1+t)^{-107 / 110}} \\
& \quad \times\left\{\delta+X(t)^{2}+X(t)^{3}+X(t)^{4}\right\}, t \geq 0,
\end{aligned}
$$

where $C$ does not depend on $\delta$ and $t$. Since the derivatives in $t$ of the second and third terms at the right hand side of (3.2) can be similarily evaluated, we finally obtain

$$
\begin{aligned}
& \sum_{j=0}^{1}(1+t)^{107 / 110}\left\|\partial_{t}^{j} n(t)\right\|_{W^{25-j, 220 / 3}} \\
& \quad \leq C\left\{\delta+X(t)^{2}+X(t)^{3}+X(t)^{4}\right\}, t \geq 0
\end{aligned}
$$

as long as the solutions $(u, n)$ of (1.1)-(1.3) exist, where $C$ does not depend on $\delta$ and $t$.

Estimates (3.52) and (3.60) complete the proof of Lemma 3.2. (Q.E.D.)

We next prove the energy estimate of the solutions $(u, n)$ to $(1.1)-(1.3)$.

LEMma 3.3. - Let $0<\varepsilon<10^{-2}$. Then, the solutions $(u, n)$ of (1.1)-(1.3) satisfy

$$
\begin{aligned}
& \sum_{j=0}^{1}\left\{\left\|\partial_{t}^{j} u(t)\right\|_{H^{52-j}}+\left\|\partial_{t}^{j} n(t)\right\|_{H^{51-j}}\right. \\
& \left.\quad+\left\|\partial_{t}^{j} n(t)\right\|_{\dot{H}^{-1-j}}\right\} \\
& \leq C\left\{\delta+X(t)^{2}\right\}, t \geq 0
\end{aligned}
$$


as long as the solutions $(u, n)$ exist. Here, $C$ does not depend on $\delta$ and $t$.

Proof. - We first derive the energy estimate of $u$. We rewrite (1.1) as the following integral equation:

$$
\begin{aligned}
u(t) & =(\cos \omega t) u_{0}+\left(\omega^{-1} \sin \omega t\right) u_{1} \\
& +\int_{0}^{t}\left(\omega^{-1} \sin \omega(t-s)\right)(n u)(s) d s, t \geq 0 .
\end{aligned}
$$

We take the $H^{52}$ norm of (3.61) to obtain by (2.30) and (2.31)

$$
\begin{aligned}
\| u(t) & \|_{H^{52}} \leq C\left(\left\|u_{0}\right\|_{H^{52}}+\left\|u_{1}\right\|_{H^{51}}\right) \\
& +C \int_{0}^{t}\|(n u)(s)\|_{H^{51}} d s \\
\leq & C \delta \\
& +C \int_{0}^{t}\left\{\|n(s)\|_{H^{51}}\|u(s)\|_{W^{25,6 /(1-2 \varepsilon)}}\right. \\
& \left.\quad+\|n(s)\|_{W^{25,220 / 3}}\|u(s)\|_{W^{26,6 /(1-2 \varepsilon)}}^{1-a}\|u(s)\|_{H^{52}}^{a}\right\} d s \\
\leq & C \delta+C \int_{0}^{t}\left\{(1+s)^{1-\varepsilon}\right. \\
& \left.+(1+s)^{-107 / 110}(1+s)^{(1-a)(1+\varepsilon)}\right\} d s \cdot X(t)^{2} \\
\leq & C \delta+C \int_{0}^{t}(1+s)^{-1-\varepsilon} d s \cdot X(t)^{2} \\
\leq & C\left\{\delta+X(t)^{2}\right\}, t \geq 0
\end{aligned}
$$

where $a=(5289-220 \varepsilon) /(5500-220 \varepsilon)$, and $C$ does not depend on $\delta$ and $t$. Since the $H^{51}$ norm of $\partial_{t} u$ can be similarily evaluated, we obtain

$$
\begin{aligned}
& \sum_{j=0}^{1}\left\|\partial_{t}^{j} u(t)\right\|_{H^{52-j}} \\
& \quad \leq C\left\{\delta+X(t)^{2}\right\}, t \geq 0
\end{aligned}
$$

as long as the solutions $(u, n)$ of (1.1)-(1.3) exist, where $C$ does not depend on $\delta$ and $t$.

The estimates of the $H^{51}$ norm of $n$ and the $H^{50}$ norm of $\partial_{t} n$ are standard ones. (3.36) and a simple calculation give us

$$
\begin{aligned}
& \sum_{j=0}^{1}\left\|\partial_{t}^{j} n(t)\right\|_{H^{51-j}} \\
& \leq C\left\{\delta+X(t)^{2}\right\}, t \geq 0,
\end{aligned}
$$


as long as the solutions $(u, n)$ of (1.1)-(1.3) exist, where $C$ does not depend on $\delta$ and $t$.

It remains only to evaluate $n$ in $\dot{H}^{-1}$ and $\partial_{t} n$ in $\dot{H}^{-2}$. We apply $\omega_{0}^{-1}$ to (3.36) and take the $L^{2}$ norm of the resulting equation to obtain by the Sobolev imbedding theorem

$$
\begin{aligned}
\left\|\omega_{0}^{-1} n(t)\right\|_{L^{2}} \leq\left\|\omega_{0}^{-1} n_{0}\right\|_{L^{2}}+\left\|\omega_{0}^{-2} n_{1}\right\|_{L^{2}} & +\int_{0}^{t}\left\|\left.u(s)\right|^{2}\right\|_{L^{2}} d s \\
\leq & C\left\{\left\|n_{0}\right\|_{\dot{H}^{-1}}+\left\|n_{1}\right\|_{\dot{H}^{-2}}\right. \\
& \left.+\int_{0}^{t}\|u(s)\|_{L^{\infty}}\|u(s)\|_{L^{2}} d s\right\} \\
\leq & C\left\{\delta+\int_{0}^{t}\|u(s)\|_{W^{1,6 /(1-2 \varepsilon)}}\|u(s)\|_{L^{2}} d s\right\} \\
\leq & C\left\{\delta+\int_{0}^{t}(1+s)^{-1-\varepsilon} d s \cdot X(t)^{2}\right\} \\
\leq & C\left\{\delta+X(t)^{2}\right\}, t \geq 0 .
\end{aligned}
$$

By differentiating (3.36) in $t$, we have

$$
\begin{aligned}
\partial_{t} n(t)=- & \left(\omega_{0} \sin \omega_{0} t\right) n_{0}+\left(\cos \omega_{0} t\right) n_{1} \\
& -\int_{0}^{t}\left(\cos \omega_{0}(t-s)\right) \omega_{0}^{2}|u(s)|^{2} d s .
\end{aligned}
$$

We apply $\omega_{0}^{-2}$ to (3.66) and take the $L^{2}$ norm of the resulting equation to obtain by the Sobolev imbedding theorem

(3.67) $\left\|\omega_{0}^{-2} \partial_{t} n(t)\right\|_{L^{2}}$

$$
\begin{aligned}
& \leq\left\|\omega_{0}^{-1} n_{0}\right\|_{L^{2}}+\left\|\omega_{0}^{-2} n_{1}\right\|_{L^{2}}+\int_{0}^{t}\left\|\left.u(s)\right|^{2}\right\|_{L^{2}} d s \\
& \leq C\left\{\left\|n_{0}\right\|_{\dot{H}^{-1}}+\left\|n_{1}\right\|_{\dot{H}^{-2}}+\int_{0}^{t}\|u(s)\|_{L^{\infty}}\|u(s)\|_{L^{2}} d s\right\} \\
& \leq C\left\{\delta+\int_{0}^{t}\|u(s)\|_{W^{1,6 /(1-2 \varepsilon)}}\|u(s)\|_{L^{2}} d s\right\} \\
& \leq C\left\{\delta+\int_{0}^{t}(1+s)^{-1-\varepsilon} d s \cdot X(t)^{2}\right\} \\
& \leq C\left\{\delta+X(t)^{2}\right\}, t \geq 0 .
\end{aligned}
$$


Therefore, (3.65) and (3.67) imply that

$$
\begin{aligned}
& \sum_{j=0}^{1}\left\|\partial_{t}^{j} n(t)\right\|_{\dot{H}^{-1-j}} \\
& \quad \leq C\left\{\delta+X(t)^{2}\right\}, t \geq 0,
\end{aligned}
$$

as long as the solutions $(u, n)$ of (1.1)-(1.3) exist, where $C$ does not depend on $\delta$ and $t$.

Accordingly, (3.63), (3.64) and (3.68) show that Lemma 3.1 holds. (Q.E.D.)

We can now easily prove Theorem 1.1 by using Lemmas 3.2 and 3.3 . Lemmas 3.2 and 3.3 yield

$$
\begin{aligned}
X(t) & \leq C\left\{\left(1+\delta+\delta^{2}\right) \delta\right. \\
& \left.+\delta X(t)+(1+\delta) X(t)^{2}+X(t)^{3}+X(t)^{4}\right\}, t \geq 0
\end{aligned}
$$

as long as the solutions $(u, n)$ of (1.1)-(1.3) exist. Here $C$ does not depend on $\delta$ and $t$. Inequality (3.69) implies that if we choose $\delta>0$ sufficiently small, then there exists an $M>0$ such that

$$
X(t) \leq M, t \geq 0 .
$$

Lemma 2.6 (ii), (iii) and (3.70) show the first claim of Theorem 1.1, that is, the unique global existence of the solutions $(u, n)$ satisfying (1.7)-(1.10) for (1.1)-(1.3).

For the proof of (1.11), it is sufficient to show that

$$
\|(n u)(t)\|_{H^{51}},\left\|\triangle|u(t)|^{2}\right\|_{H^{50}} \in L^{1}(0, \infty) .
$$

Now that we have (3.70), this is essentially proved in the proof of Lemma 3.3 (see, e.g., (3.62)). We omit the proof of (1.11).

(Q.E.D.)

We finally state the proof of Corollary 1.2. Corollary 1.2 is an immediate consequence of Theorem 1.1 and Lemma 2.6 (ii).

Concluding remark. - Recently, Sideris [13] and Georgiev [7] have proved the good decay estimates of solution for the inhomogeneous linear Klein-Gordon equation by using the fundamental solution of the linear Klein-Gordon equation and the generators of the Lorentz group. However, the authors do not know whether one can prove Theorem 1.1 by using the decay estimates in [13] and [7] instead of the method of normal form. 


\section{REFERENCES}

[1] A. BaChelot, Problème de Cauchy globale pour des système de Dirac-Klein-Gordon, Ann. Inst. Henri Poincaré, Phys. Théor., Vol. 48, 1988, pp. 387-422.

[2] J. BERGH and J. LÖFSTRÖM, Interpolation Spaces, Springer-Verlag, Berlin-Heidelberg-New York, 1976.

[3] H. BrÉzIS and T. GallouËT, Non linear Schrödinger evolution equations, Nonlinear Analysis, TMA, Vol. 4, 1980, pp. 677-681.

[4] A. Friedman, Partial Differential Equations, Holt Rinehart and Winston, New York, 1969.

[5] V. GeorGiev, Global solutions of the system of wave and Klein-Gordon equations, Math. Z., Vol. 203, 1990, pp. 683-698.

[6] V. Georgiev, Small amplitude solutions of the Maxwell-Dirac equations, Indiana Univ. Math. J., Vol. 40, 1991, pp. 845-883.

[7] V. Georgiev, Decay estimates for the Klein-Gordon equations, Commun. Part. Diff. Eqs., Vol. 17, 1992, pp. 1111-1139.

[8] J. GinIBRE and G. VELo, Time decay of finite energy solutions of the non linear KleinGordon and Schrödinger equations, Ann. Inst. Henri Poincaré, Phys. Théor., Vol. 43, 1985, pp. 399-442.

[9] S. KLAINERMAN, Uniform decay estimates and the Lorentz invariance of the classical wave equations, Comm. Pure Appl. Math., Vol. 38, 1985, pp. 321-332.

[10] S. Klainerman, The null condition and global existence to nonlinear wave equations, Lect. in Appl. Math., Vol. 23, 1986, pp. 293-326.

[11] H. PECHER, Nonlinear small data scattering for the wave and Klein-Gordon equation, Math. Z., Vol. 185, 1984, pp. 261-270.

[12] J. Shatah, Normal forms and quadratic nonlinear Klein-Gordon equations, Comm. Pure Appl. Math., Vol. 38, 1985, pp. 685-696.

[13] T. SDERIS, Decay estimates for the three-dimensional inhomogeneous Klein-Gordon equation and applications, Commun. Part. Diff. Egs., Vol. 14, 1989, pp. 1421-1455.

[14] W. A. Strauss, Nonlinear Wave Equations, CBMS Regional Conference Series in Mathematics, no. 73, Amer. Math. Soc., Providence, RI, 1989.

[15] S. G. ThornhnL and D. ter HAAR, Langmuir turbulence and modulational instability, Phys. Reports (Sect. C of Phys. Lett.), Vol. 43, 1978, pp. 43-99.

[16] J. C. H. SimON and E. TAFLIN, The Cauchy problem for non-linear Klein-Gordon equations, Commun. Math. Phys., Vol. 152, 1993, pp. 433-478.

(Manuscript received February 16, 1994.)

Vol. $12, n^{\circ} 4-1995$. 